\title{
Coronary atherosclerosis: Un-altered physiology
}

2

3 Paul de Groot MD PhD ${ }^{1^{*}}$, Roel W Veldhuizen MD PhD ${ }^{2}$

4

$5 \quad{ }^{1}$ Formerly Department of Cardiology, Haaglanden MC, The Hague, the Netherlands

$6 \quad{ }^{2}$ Formerly Department of Pathology, Haaglanden MC, The Hague, the Netherlands

7

8

* Correspondence:

9 Dr. Paul de Groot

Wijkselaan 6,2554GE The Hague, the Netherlands. E-mail: wijkse6@gmail.com 


\section{Abstract}

Background: End-stage coronary artery atherosclerosis has been studied extensively but the exact mechanisms of initiation and progression have not been defined fully. The aim of this study was to mathematically describe luminal change in relation to coronary vessel wall thickness in its progression from normal to atherosclerotic to establish whether these explain the pathophysiology.

Methods: One hundred coronary artery sections were graded histologically as 'normal' to 'highly atherosclerotic'. Random systemic sampling by image analysis yielded 32 measurements (lumen radius and intima, medial, and adventitial thickness) from each section along 32 evenly spaced radii.

Results: The raw data follow an undulating course in relation to successive segments in all sections analyzed, pointing to a dynamic and well-ordered system. The calculated values, studied in triplets, followed a non-synchronized parabolic course, which was converted to linearity by taking the change in numbers $(n-(n-1)=\Delta)$ into account. The course and sign of ' $\Delta$ vessel wall' (resulting from summed $\Delta$ intima, $\Delta$ media, $\Delta$ adventitia) and ' $\Delta$ lumen radius' values were unique for each triplet. Triplets order according to ' $\Delta$ lumen radius minus $\Delta$ vessel wall' and its course given by the trendline a-value presented stages in which increased $\Delta$ vessel wall resulted in increased $\Delta$ lumen radius in stages 1 and 3 and decreased $\Delta$ lumen radius in stage 2 . This phenomenon was found in all sections regardless of histological indication and independent of vessel wall constituent parts (intima, media, adventitia).

Conclusions: Similar basic processes are defined in all sections regardless of histological rating, indicating un-altered physiology. As such, coronary atherosclerosis can only be defined by a large to small shift of the triplets $\Delta$ vessel wall trendline a-value. Consequently, 
no parameter of vessel wall pathology exists in absolute terms. Vessel wall composition has no importance for $\Delta$ lumen radius.

38 Keywords: coronary artery, atherosclerosis, image analysis, basic process, triplets course, semi-ranges 


\section{Introduction}

The anatomy and pathology of the coronary arteries are widely studied, especially by histological means. However, basic knowledge is lacking regarding, for example, the mechanism of arterial tapering, which corresponds to deficient knowledge of vessel wall/lumen interactions and, even more fundamentally, the intimal, medial, adventitial interplay.

Research has traditionally focused on the histopathology of plaque formation, the end-stage atherosclerotic process. The process from initiation to plaque formation has often been studied in a retrograde manner [1-3], which may lead to cause/effect reversal. An enlarged intima was considered a characteristic and regarded as a key player in atherosclerotic pathology $[4,5]$. Consequently, the limits of normality, the thickness of the media and adventitia, and their role in atherosclerosis remain elusive.

We hypothesized that mathematical differences in the cross-sectional dimensions of normal and atherosclerotic arteries between stages could be used to help explain the pathophysiology of coronary artery atherosclerosis. This study aims to bridge the knowledge gap between the physiology of vessel morphology and the pathogenesis of atherosclerosis by mathematically defining both processes. Using a specially designed morphometric method termed 'random systemic sampling' [6], we aim to define how vessel wall thickness and lumen size are related and how the vessel wall layers (intima, media, adventitia) contribute to the pathophysiology of atherosclerosis.

\section{Material and Methods}

\section{Study population and ethical approval}


Study samples were obtained from autopsies of unselected patients regardless of the cause of death. No inclusion or exclusion criteria were applied. A total 100 cases were accepted and included for study. The Haaglanden MC ethics committee approved the study protocol and the use of specimens obtained from routine post-mortem examinations.

\section{Coronary artery sampling and histology}

Tissue blocks from the heart with coronary vessels attached were fixed by submersion in $4 \%$ phosphate-buffered formaldehyde solution $(0.1 \mathrm{M}, \mathrm{pH} 7.0)$ for 48 hours prior to further sampling. The arteries were not pressure perfused in order to retain 'natural' residual vascular wall stress. Vessel cross-sections were selected at random with no preference for left or right coronary arteries, sampling site, or cause of death. Sections were processed using routine methods, paraffin embedded, and 5- $\mu$ m sections cut and stained with hematoxylin and eosin for microscopic assessment. Morphometric assessment was preferentially performed on von Gieson/elastica stained sections.

\section{Dataset}

All sections were imaged and assessed by microscopy. Sections were histologically defined as follows: normal (intima < media), circumferential non-atherosclerotic enlarged vessel wall (intima $\geq$ media), circumferential and unilateral atherosclerotic enlarged vessel wall (signs of atherosclerosis, i.e., pattern of acellularity, cholesterol deposition, calcification, fibrosis, inflammation). Thus, all sections together represented the course from 'normal' (no signs of atherosclerosis) to 'circumferential atherosclerosis'. One fully analyzed specimen from each histologically defined group highlighted the mathematical changes during progression. An additional section was histologically rated as 'extreme unilateral atherosclerosis' (not shown).

\section{Image analysis}


Computer-assisted image analysis was performed using ZEN 2 lite software (Carl Zeiss).

Digitized tissue sections were displayed on a monitor and the center of each vessel located using a computerized best fit circle procedure. Only sections with an external elastic lamina Feret circle of at least 0.8 were used to exclude sectioning artefacts and interpretation bias.

Using a random starting position, 32 radii were drawn from the center (i.e., one every 11.25 degrees) (random systemic sampling; Fig 1). The observer then identified and marked the positions of the endothelium, internal and external elastic lamina, and the adventitial-fat border along each radius. The lumen radius (Rlu) and the dimensions of the intima, media, and adventitia (INTd, MEDd, and ADVd, respectively) at each location were calculated in micrometers by the software. Matched values along each radius were jointly termed the 'measurement unit'. Thirty-two measurement units were available for each section. All data were tabulated for further analysis.

Fig 1. Random systemic sampling computer-assisted image analysis.

The center point is located by the best fit circle procedure. Thirty-two radii differing by 11.25 degrees defined 32 measurement units of the lumen radius and intima, media, and adventitia dimensions.

\section{Validation of the term value as a computerized classification parameter}

INTd, MEDd, and ADVd were highly variable, even between two consecutive measurement units and regardless of the originating section. As the three vessel wall layer dimensions were not usually independent, they were defined as a single functional unit (FU-1). Similarly, RluWd, VWd, and Rlu defined a functional unit (FU-2). The relationship between the three values constituting FU-1 and FU-2 was parabolic and interrelated according to the formula $a x^{2}+b x+c$. A constant characteristic of each functional unit (the 'term value'; T-FU1 and T- 
FU2) was produced by subtracting the difference between ADVd and MEDd from the difference between MEDd and INTd, and subtracting Rlu-VWd from VWd-(Rlu-VWd), respectively.

\section{Process study}

Data from random systemic sampling represented a static state, a snapshot in time. Each segment provided FU-1 and FU-2, which were characterized by T-FU1 and T-FU2, respectively. T-FU2 values set against consecutive segment numbers showed a well-ordered saw-toothed course in each section; the upward course changed to a downward course at an acute angle (Fig 2-5), indicating a dynamic process.

\section{Fig 2. Section rated 'normal'.}

(A) Micrograph. (B) Scatter diagram T-FU2, Rlu-VWd, VWd, and Rlu vs. segment numbers. (C) TFU1, INTd, MEDd, and ADVd vs. segment numbers.

Fig 3. Section rated 'circumferential enlarged intima'.

(A) Micrograph. (B) Scatter diagram T-FU2, Rlu-VWd, VWd, and Rlu vs. segment numbers. (C) TFU1, INTd, MEDd, and ADVd vs. segment numbers.

\section{Fig 4. Section rated 'unilateral atherosclerosis'.}

(A) Micrograph. (B) Scatter diagram T-FU2, Rlu-VWd, VWd, and Rlu vs. segment numbers. (C) TFU1, INTd, MEDd, and ADVd vs. segment numbers.

\section{Fig 5. Section rated 'circumferential atherosclerosis'.}

(A) Micrograph. (B) Scatter diagram T-FU2, Rlu-VWd, VWd, and Rlu vs. segment numbers. (C) TFU1, INTd, MEDd, and ADVd vs. segment numbers. 
The changes in the INTd, MEDd, and ADVd and Rlu-VWd, VWd, and Rlu of consecutive segments were studied in triplets. Each triplet provided three successive values of FU-1 and FU-2 defined by their collective parabolic course and represented by their corresponding trendline formula $\left(\mathrm{R}^{2}=1\right)$. Intermediate segment values were calculated by interpolation. For example, triplet (1-3): segment numbers $(\mathrm{x})$ and segment numbers squares $\left(\mathrm{x}^{2}\right)$ interpolated in formula $\mathrm{y}=\mathrm{ax}^{2}+\mathrm{bx}+\mathrm{c}$ provided calculated $\mathrm{y}$ values of $\mathrm{x}=1 ; 1.1 ; 1.2 ; \ldots 2 ; 2.1 ; \ldots 3$.

Therefore, each section provided 16 triplets $(1-3),(3-5), \ldots$ (31-1), each consisting of 20 calculated FU-1 and FU-2 component values. The non-synchronized parabolic course of INTd, MEDd, and ADVd and Rlu-VWd, VWd, and Rlu in relation to T-FU1 and T-FU2, respectively, were marked by strong variability in peak/bottom parabola values, which made mutual comparisons impossible. Therefore, a step by step course was described by the value change ( $\Delta$ values 1.1-1.0 $\ldots \mathrm{n}$-(n-1)), which transformed the parabolic relationship into a linear one (trendline formula: $\mathrm{y}=\mathrm{ax}+\mathrm{b}, \mathrm{R}^{2}=1$ ).

\section{Results}

\section{Measurement data}

Measurement data for 'normal' (Table 1, Fig 2), 'circumferentially enlarged intima' (Table 2, Fig 3), 'unilateral atherosclerosis' (Table 3, Fig 4), and 'circumferential atherosclerosis' sections (Table 4, Fig 5) showed the saw-toothed course of T-FU2 in relation to segment number. The additional 'extreme unilateral atherosclerosis' section (not shown) also presented this phenomenon. T-FU2 curves were similar to Rlu-VWd curves, and to a lesser extent Rlu curves. The VWd and Rlu were generally antagonistic, but some segments followed a congruent course. An increase in the VWd was not always accompanied by a decrease in Rlu, and vice versa. The INTd, MEDd, and ADVd also alternated, but did not follow a distinct pattern. 
Table 1. Measurement Values of 32 Segments in a 'Normal' Section.

\begin{tabular}{|c|c|c|c|c|c|c|c|c|}
\hline Segment & T-FU2 & Rlu-VWd & VWd & Rlu & T-FU1 & INTd & MEDd & AVDd \\
\hline 1 & 181 & 143 & 105 & 248 & -68 & 3 & 58 & 45 \\
\hline 2 & 32 & 85 & 138 & 223 & 5 & 18 & 44 & 76 \\
\hline 3 & 68 & 97 & 126 & 223 & -109 & 7 & 79 & 41 \\
\hline 4 & -2 & 59 & 121 & 181 & -34 & 13 & 52 & 57 \\
\hline 5 & -52 & 41 & 135 & 176 & -6 & 13 & 47 & 75 \\
\hline 6 & -62 & 36 & 133 & 168 & -7 & 13 & 47 & 74 \\
\hline 7 & -138 & 10 & 158 & 168 & -6 & 6 & 55 & 97 \\
\hline 8 & -25 & 54 & 132 & 185 & -17 & 10 & 50 & 72 \\
\hline 9 & -25 & 54 & 133 & 186 & -14 & 13 & 49 & 71 \\
\hline 10 & -2 & 67 & 136 & 203 & -17 & 9 & 51 & 76 \\
\hline 11 & -11 & 66 & 143 & 208 & -69 & 13 & 70 & 59 \\
\hline 12 & 72 & 97 & 123 & 220 & -9 & 18 & 44 & 61 \\
\hline 13 & 141 & 132 & 122 & 254 & 0 & 13 & 41 & 69 \\
\hline 14 & 139 & 130 & 121 & 251 & -28 & 11 & 50 & 60 \\
\hline 15 & 150 & 134 & 119 & 253 & -48 & 11 & 56 & 52 \\
\hline 16 & 155 & 134 & 113 & 247 & -49 & 7 & 54 & 52 \\
\hline 17 & 124 & 128 & 131 & 259 & -42 & 16 & 58 & 58 \\
\hline 18 & 190 & 152 & 115 & 267 & -22 & 20 & 46 & 49 \\
\hline 19 & 133 & 129 & 126 & 256 & -35 & 20 & 54 & 53 \\
\hline 20 & 147 & 137 & 128 & 265 & -27 & 16 & 52 & 60 \\
\hline 21 & 62 & 100 & 138 & 238 & -60 & 13 & 66 & 60 \\
\hline 22 & -30 & 62 & 154 & 216 & -25 & 27 & 60 & 68 \\
\hline 23 & 2 & 72 & 142 & 214 & -31 & 14 & 58 & 70 \\
\hline 24 & -80 & 37 & 154 & 190 & -23 & 16 & 59 & 79 \\
\hline 25 & -107 & 26 & 160 & 186 & 7 & 20 & 51 & 89 \\
\hline 26 & -59 & 39 & 136 & 175 & -44 & 10 & 60 & 66 \\
\hline 27 & -39 & 43 & 125 & 168 & -74 & 10 & 66 & 49 \\
\hline 28 & -31 & 47 & 125 & 172 & -67 & 3 & 64 & 58 \\
\hline 29 & 22 & 69 & 116 & 185 & -63 & 9 & 60 & 47 \\
\hline 30 & 35 & 81 & 128 & 209 & -64 & 19 & 64 & 45 \\
\hline 31 & 121 & 119 & 116 & 235 & -10 & 10 & 42 & 65 \\
\hline 32 & -13 & 70 & 153 & 223 & 0 & 13 & 51 & 89 \\
\hline
\end{tabular}

163

Segment clusters comprise 1-7, 8-17, 18-28, and 19-32. 
Table 2. Measurement Values of 32 Segments in a 'Circumferential Enlarged Intima' Section.

\begin{tabular}{|c|c|c|c|c|c|c|c|c|}
\hline Segment & T-FU2 & r-lu-VW & VW-dik & r-lumen & T-FU1 & intdik & meddik & advdik \\
\hline 1 & -608 & 53 & 713 & 766 & 194 & 252 & 173 & 288 \\
\hline 2 & -462 & 114 & 690 & 804 & 116 & 244 & 191 & 254 \\
\hline 3 & -349 & 171 & 690 & 861 & 92 & 230 & 200 & 261 \\
\hline 4 & -370 & 180 & 730 & 910 & 96 & 238 & 211 & 280 \\
\hline 5 & -171 & 270 & 711 & 981 & 67 & 226 & 215 & 270 \\
\hline 6 & 29 & 363 & 697 & 1060 & 128 & 223 & 190 & 284 \\
\hline 7 & 157 & 426 & 696 & 1123 & 57 & 204 & 213 & 280 \\
\hline 8 & 95 & 410 & 725 & 1135 & 235 & 268 & 163 & 294 \\
\hline 9 & 407 & 523 & 638 & 1161 & 74 & 225 & 188 & 225 \\
\hline 10 & 657 & 620 & 583 & 1203 & 96 & 211 & 162 & 210 \\
\hline 11 & 701 & 631 & 562 & 1193 & 55 & 166 & 169 & 227 \\
\hline 12 & 435 & 515 & 594 & 1109 & 94 & 160 & 167 & 268 \\
\hline 13 & 368 & 461 & 554 & 1015 & 41 & 172 & 171 & 211 \\
\hline 14 & 58 & 335 & 611 & 946 & 65 & 164 & 182 & 265 \\
\hline 15 & 114 & 338 & 562 & 899 & 3 & 172 & 186 & 204 \\
\hline 16 & -101 & 249 & 599 & 847 & 45 & 197 & 185 & 217 \\
\hline 17 & -342 & 155 & 652 & 808 & 243 & 300 & 136 & 216 \\
\hline 18 & -491 & 109 & 708 & 817 & 345 & 317 & 121 & 270 \\
\hline 19 & -380 & 156 & 693 & 849 & 299 & 280 & 131 & 281 \\
\hline 20 & -563 & 103 & 769 & 872 & 250 & 297 & 173 & 300 \\
\hline 21 & -529 & 136 & 801 & 937 & 332 & 293 & 156 & 352 \\
\hline 22 & -489 & 172 & 833 & 1005 & 376 & 272 & 152 & 408 \\
\hline 23 & 63 & 377 & 691 & 1068 & 355 & 287 & 112 & 292 \\
\hline 24 & 452 & 544 & 637 & 1181 & 298 & 268 & 113 & 256 \\
\hline 25 & 978 & 750 & 521 & 1270 & 79 & 194 & 147 & 180 \\
\hline 26 & 718 & 656 & 594 & 1250 & 160 & 252 & 145 & 197 \\
\hline 27 & 433 & 538 & 643 & 1181 & 316 & 245 & 109 & 289 \\
\hline 28 & 315 & 463 & 611 & 1074 & 243 & 237 & 123 & 252 \\
\hline 29 & 70 & 358 & 646 & 1004 & 301 & 227 & 115 & 303 \\
\hline 30 & -126 & 258 & 643 & 901 & 219 & 227 & 141 & 274 \\
\hline 31 & -357 & 158 & 673 & 830 & 222 & 235 & 150 & 288 \\
\hline 32 & -554 & 80 & 714 & 794 & 222 & 235 & 164 & 315 \\
\hline
\end{tabular}

166

Segment clusters comprise 1-10, 11-14, 15-18, 19-22, 23-25, and 26-32. 
Table 3. Measurement Values of 32 Segments in a 'Unilateral Atherosclerosis' Section.

\begin{tabular}{|c|c|c|c|c|c|c|c|c|}
\hline Segment & T-FU2 & rlu-VW & VWdik & Rlu & T-FU1 & INTd & MEDd & $A D V d$ \\
\hline 1 & -1528 & -469 & 590 & 121 & 483 & 433 & 36 & 121 \\
\hline 2 & -1608 & -498 & 611 & 112 & 519 & 464 & 31 & 116 \\
\hline 3 & -1741 & -543 & 655 & 113 & 576 & 493 & 26 & 136 \\
\hline 4 & -1823 & -569 & 685 & 116 & 545 & 499 & 47 & 140 \\
\hline 5 & -1671 & -509 & 653 & 145 & 477 & 497 & 59 & 98 \\
\hline 6 & -913 & -201 & 511 & 311 & 334 & 357 & 59 & 96 \\
\hline 7 & -356 & 28 & 412 & 440 & 219 & 240 & 64 & 107 \\
\hline 8 & -199 & 100 & 399 & 499 & 245 & 207 & 51 & 141 \\
\hline 9 & 260 & 286 & 312 & 598 & 139 & 118 & 58 & 136 \\
\hline 10 & 215 & 263 & 311 & 574 & 172 & 92 & 46 & 172 \\
\hline 11 & 156 & 231 & 306 & 537 & 146 & 83 & 54 & 169 \\
\hline 12 & 163 & 228 & 292 & 520 & 119 & 61 & 58 & 173 \\
\hline 13 & 310 & 272 & 234 & 506 & 63 & 55 & 57 & 122 \\
\hline 14 & 309 & 276 & 244 & 520 & 94 & 48 & 50 & 147 \\
\hline 15 & 462 & 328 & 194 & 522 & 48 & 44 & 49 & 101 \\
\hline 16 & 220 & 234 & 247 & 481 & 119 & 73 & 43 & 131 \\
\hline 17 & 207 & 227 & 247 & 474 & 58 & 76 & 63 & 108 \\
\hline 18 & 141 & 202 & 263 & 465 & 76 & 81 & 62 & 119 \\
\hline 19 & 91 & 180 & 270 & 450 & 62 & 75 & 69 & 126 \\
\hline 20 & 4 & 145 & 286 & 432 & 37 & 77 & 83 & 127 \\
\hline 21 & -1 & 146 & 292 & 438 & 73 & 78 & 73 & 141 \\
\hline 22 & 112 & 195 & 277 & 472 & 93 & 86 & 61 & 130 \\
\hline 23 & 183 & 231 & 280 & 511 & 113 & 68 & 56 & 157 \\
\hline 24 & 192 & 246 & 301 & 547 & 169 & 81 & 44 & 177 \\
\hline 25 & 59 & 208 & 358 & 566 & 169 & 71 & 63 & 224 \\
\hline 26 & -113 & 126 & 365 & 491 & 119 & 113 & 82 & 170 \\
\hline 27 & -449 & -11 & 427 & 415 & 207 & 170 & 73 & 184 \\
\hline 28 & -383 & -1 & 381 & 380 & 212 & 189 & 56 & 135 \\
\hline 29 & -578 & -87 & 404 & 317 & 234 & 246 & 57 & 102 \\
\hline 30 & -850 & -200 & 450 & 249 & 314 & 311 & 45 & 93 \\
\hline 31 & -1224 & -347 & 529 & 182 & 428 & 368 & 34 & 127 \\
\hline 32 & -1286 & -381 & 524 & 143 & 432 & 391 & 31 & 102 \\
\hline
\end{tabular}

170 Segment clusters comprise 1-4, 5-8, 9-11, 12-15, 16-21, 22-24, and 25-32. 
Table 4. Measurement Values of 32 Segments in a 'Circumferential Atherosclerosis' Section.

\begin{tabular}{ccccccccc}
\hline Segment & T-FU2 & rlu-VW & VWdik & Rlu & T-FU1 & INTd & MEDd & ADVd \\
\hline 1 & $\mathbf{- 1 9 7 2}$ & -493 & 986 & 493 & $\mathbf{7 1 8}$ & 724 & 89 & 173 \\
2 & $\mathbf{- 2 2 0 1}$ & -573 & 1055 & 482 & $\mathbf{6 3 9}$ & 718 & 139 & 198 \\
3 & $\mathbf{- 2 3 1 5}$ & -609 & 1097 & 488 & $\mathbf{6 3 4}$ & 707 & 154 & 236 \\
4 & $\mathbf{- 2 4 7 0}$ & -654 & 1162 & 507 & $\mathbf{6 6 5}$ & 764 & 166 & 232 \\
5 & $\mathbf{- 2 5 7 8}$ & -674 & 1230 & 555 & $\mathbf{7 6 3}$ & 785 & 156 & 289 \\
6 & $\mathbf{- 2 6 1 2}$ & -668 & 1275 & 607 & $\mathbf{8 1 8}$ & 780 & 153 & 343 \\
7 & $\mathbf{- 2 3 5 1}$ & -561 & 1228 & 666 & $\mathbf{7 9 1}$ & 774 & 146 & 308 \\
8 & $\mathbf{- 2 0 8 1}$ & -448 & 1185 & $\mathbf{7 3 6}$ & $\mathbf{7 4 4}$ & 760 & 147 & 278 \\
9 & $\mathbf{- 1 7 9 6}$ & -325 & 1146 & 821 & $\mathbf{7 3 8}$ & 721 & 136 & 288 \\
10 & $\mathbf{- 2 3 8}$ & 286 & 810 & 1096 & $\mathbf{3 7 6}$ & 383 & 145 & 283 \\
11 & $\mathbf{- 4 6 6}$ & 185 & 837 & 1022 & $\mathbf{4 8 4}$ & 450 & 118 & 269 \\
12 & $\mathbf{- 9 1 1}$ & 2 & 916 & 919 & $\mathbf{5 4 2}$ & 472 & 125 & 319 \\
13 & $\mathbf{- 1 5 8 5}$ & -283 & 1020 & $\mathbf{7 3 7}$ & $\mathbf{6 9 3}$ & 600 & 109 & 311 \\
14 & $\mathbf{- 1 7 6 5}$ & -377 & 1012 & 635 & $\mathbf{5 4 5}$ & 575 & 156 & 281 \\
15 & $\mathbf{- 2 1 4 3}$ & -543 & 1057 & 514 & $\mathbf{6 5 4}$ & 657 & 134 & 266 \\
16 & $\mathbf{- 2 2 9 3}$ & -614 & 1065 & 450 & $\mathbf{5 0 3}$ & 665 & 187 & 213 \\
17 & $\mathbf{- 2 4 6 4}$ & -682 & 1101 & 420 & $\mathbf{6 4 5}$ & 739 & 152 & 210 \\
18 & $\mathbf{- 2 7 1 3}$ & -774 & 1164 & 390 & $\mathbf{7 3 2}$ & 796 & 144 & 224 \\
19 & $\mathbf{- 3 0 5 6}$ & -893 & 1271 & 378 & $\mathbf{9 0 8}$ & 836 & 121 & 314 \\
20 & $\mathbf{- 3 5 5 3}$ & -1057 & 1438 & 381 & $\mathbf{9 4 1}$ & 882 & 166 & 390 \\
21 & $\mathbf{- 3 6 6 1}$ & -1086 & 1489 & 404 & $\mathbf{1 0 4 4}$ & 907 & 148 & 434 \\
22 & $\mathbf{- 3 7 0 4}$ & -1080 & 1544 & 464 & $\mathbf{1 0 9 2}$ & 923 & 151 & 470 \\
23 & $\mathbf{- 3 3 1 0}$ & -926 & 1459 & 533 & $\mathbf{1 0 6 5}$ & 861 & 131 & 467 \\
24 & $\mathbf{- 2 1 4 9}$ & -480 & 1189 & 709 & $\mathbf{8 6 5}$ & 702 & 108 & 378 \\
25 & $\mathbf{- 1 9 3 6}$ & -393 & 1151 & $\mathbf{7 5 8}$ & $\mathbf{9 1 6}$ & $\mathbf{7 4 2}$ & $\mathbf{7 8}$ & 330 \\
26 & -1108 & -65 & 978 & 913 & $\mathbf{7 7 1}$ & 591 & 69 & 318 \\
27 & -1008 & -38 & 931 & 893 & $\mathbf{7 1 3}$ & 573 & 73 & 285 \\
28 & $\mathbf{- 1 5 1 7}$ & -269 & 978 & 708 & $\mathbf{8 3 7}$ & 667 & 47 & 264 \\
29 & $\mathbf{- 1 4 5 0}$ & -257 & 935 & 678 & $\mathbf{7 3 4}$ & 604 & 67 & 264 \\
30 & $\mathbf{- 1 5 3 9}$ & -304 & 930 & 626 & $\mathbf{7 3 3}$ & 629 & 66 & 235 \\
31 & $\mathbf{- 1 6 9 4}$ & -374 & 946 & 571 & $\mathbf{6 7 7}$ & 657 & 90 & 199 \\
32 & $\mathbf{- 1 9 3 5}$ & -467 & 1002 & 535 & $\mathbf{7 2 3}$ & 708 & 93 & 201 \\
\hline
\end{tabular}

174 Segment clusters comprise 1-6, 7-9, 10-19, 20-27, and 28-32.

175

176 Triplets

177 Each section consisted of 16 triplets, and each triplet consisted of 20 associated data points.

178 After data conversion to linear courses $(\Delta)$, various triplets showed that $\Delta$ T-FU2 and $\Delta$ Rlu- 
VWd ( $\mathrm{R}^{2}$ always $\left.>0.95\right)$ alternated from increasing to decreasing order. Regardless of a section's histological rating, nine triplets showed a decrease and seven triplets an increase. Increasing and decreasing triplets were not always successive.

The paths followed by $\Delta \mathrm{Rlu}-\mathrm{VWd}, \Delta \mathrm{VWd}, \Delta \mathrm{Rlu}, \Delta \mathrm{INTd}, \Delta \mathrm{MEDd}$, and $\Delta \mathrm{ADVd}$ within each triplet were unique and differed in various triplets. Marks were discerned within several triplets (asynchronous, course-dependent, section-independent): $\Delta \mathrm{Rlu}-\mathrm{VWd} \approx 0$ resulted in $\Delta \mathrm{VWd} \approx \Delta \mathrm{Rlu}, \Delta \mathrm{VWd} \approx 0$ gives $\Delta \mathrm{Rlu}-\mathrm{VWd} \approx \Delta \mathrm{Rlu}$, and $\Delta \mathrm{Rlu} \approx 0$ resulted in $\Delta \mathrm{VWd} \approx-\Delta \mathrm{Rlu}-$ VWd. Asynchronous $\triangle \mathrm{INTd}, \triangle \mathrm{MEDd}$, or $\triangle \mathrm{ADVd} \approx 0$ was also encountered without noticeable influence on the $\Delta \mathrm{VWd}$ sequence. Even two of these being $\approx 0$ did not influence the $\Delta \mathrm{VWd}$ course. Marks in various triplets were not accompanied by $\Delta \mathrm{T}-\mathrm{FU} 2$ or $\Delta \mathrm{Rlu}-\mathrm{VWd}$ similarity.

\section{Triplet interaction}

The $\Delta \mathrm{VWd}, \Delta \mathrm{Rlu}, \Delta \mathrm{INTd}, \Delta \mathrm{MEDd}$, and $\triangle \mathrm{ADVd}$ values of all triplets originating from each section were set against $\Delta \mathrm{Rlu}-\mathrm{VWd}$ separately, resulting in summed trendline a-values for $\Delta \mathrm{VWd}$ and $\Delta \mathrm{Rlu}=1$ (Table 5). Consecutive triplets did not constitute a systemic sequence, but ordering in descending order based on $\Delta \mathrm{VWd}$ trendline a-values obtained a regular sequence. Three stages were observed: stage 1 marked by trendline a-values $\Delta \mathrm{VWd}$ and $\Delta \mathrm{Rlu}$ $>0$; stage 2 marked by antagonistic a-values $\Delta \mathrm{VWd}$ and $\Delta \mathrm{Rlu}$; stage 3 marked by a-values $\Delta \mathrm{VWd}$ and $\Delta \mathrm{Rlu}<0$. Transition stage 1 to stage 2 was marked by a-value $\Delta \mathrm{VWd}=0$ and by a-value $\Delta$ Rlu $=1$, whereas transition from stage 2 to stage 3 presented the opposite (i.e., avalue $\Delta \mathrm{VWd}=1$ and a-value $\Delta \mathrm{Rlu}=0$ ). All sections showed three stages except 'extreme unilateral atherosclerosis', which lacked stage 1 due to a $\Delta \mathrm{VWd}$ trendline a-value shift from stage 1 to stage 3 . 


\section{Normal}

\begin{tabular}{|c|c|c|c|c|c|c|}
\hline & & $\Delta \mathrm{VWd}$ & $\Delta$ Rlu & $\triangle \mathrm{INTd}$ & $\triangle \mathrm{MEDd}$ & $\triangle \mathrm{ADVd}$ \\
\hline \multirow[t]{2}{*}{ stage 1} & $(11-13)$ & $y=7.6157 x-26.171$ & $y=8.6159 x-26.171$ & $y=-3.6986 x+12.18$ & $y=8.9594 x-31.075$ & $y=2.3548 x-7.2757$ \\
\hline & $(3-5)$ & $y=0.9273 x+3.009$ & $y=1.9273 x+3.0086$ & $y=-0.2813 x-0.5078$ & $y=1.1283 x+1.5693$ & $y=0.0803 x+1.9477$ \\
\hline \multirow[t]{11}{*}{ stage 2} & $(19-21)$ & $y=-0.1983 x+0.3041$ & $y=0.8017 x+0.3037$ & $y=-0.0281 x-0.4178$ & $y=-0.3569 x+0.094$ & $y=0.1867 x+0.6283$ \\
\hline & $(23-25)$ & $y=-0.2155 x+0.3957$ & $y=0.7845 x+0.3942$ & $y=0.1094 x+0.5529$ & $y=-0.3642 x-1.1637$ & $y=0.0393 x+1.0061$ \\
\hline & $(13-15)$ & $y=-0.2152 x-0.1536$ & $y=0.7849 x-0.1533$ & $y=0.2189 x-0.0907$ & $y=-0.4797 x+0.7966$ & $y=0.0456 x-0.8595$ \\
\hline & $(9-11)$ & $y=-0.263 x+0.6603$ & $y=0.737 x+0.6615$ & $y=-0.583 x+0.3723$ & $y=-1.1775 x+1.7978$ & $y=1.4974 x-1.509$ \\
\hline & $(27-29)$ & $y=-0.4643 x+0.1325$ & $y=0.5357 x+0.1325$ & $y=0.7248 x-0.9672$ & $y=-0.1049 x-0.2067$ & $y=-1.0842 x+1.3059$ \\
\hline & $(21-23)$ & $y=-0.5926 x-0.6261$ & $y=0.4074 x-0.6292$ & $y=-0.5616 x-0.711$ & $y=0.0781 x-0.3047$ & $y=-0.1092 x+0.3874$ \\
\hline & $(17-19)$ & $y=-0.5944 x-0.2178$ & $y=0.4056 x-0.2178$ & $y=0.086 x+0.198$ & $y=-0.4227 x-0.1787$ & $y=-0.2578 x-0.234$ \\
\hline & $(7-9)$ & $y=-0.6364 x+0.1033$ & $y=0.3636 x+0.1027$ & $y=0.0146 x+0.301$ & $y=-0.1011 x-0.0792$ & $y=-0.5498 x-0.1188$ \\
\hline & $(1-3)$ & $y=-0.6428 x-0.424$ & $y=0.3572 x-0.4247$ & $y=-0.3765 x-0.6698$ & $y=0.6775 x+2.5999$ & $y=-0.9437 x-2.3548$ \\
\hline & $(31-1)$ & $y=-0.7474 x-0.0235$ & $y=0.2526 x-0.0235$ & $y=-0.0501 x+0.0185$ & $y=-0.1982 x-0.0682$ & $y=-0.499 x+0.0259$ \\
\hline & $(29-31)$ & $y=-0.9225 x+2.3182$ & $y=0.0775 x+2.319$ & $y=-0.7419 x+1.871$ & $y=-1.0645 x+1.7754$ & $y=0.884 x-1.3226$ \\
\hline \multirow[t]{3}{*}{ stage 3} & $(5-7)$ & $y=-1.3865 x-0.9985$ & $y=-0.3864 x-0.9985$ & $y=0.3197 x+0.1872$ & $y=-0.4367 x-0.2977$ & $y=-1.2694 x-0.8887$ \\
\hline & $(25-27)$ & $y=-1.6533 x-0.3501$ & $y=-0.6532 x-0.3507$ & $y=-1.3156 x+0.5848$ & $y=0.3858 x+0.4492$ & $y=-0.7234 x-1.3847$ \\
\hline & $(15-17)$ & $y=-4.2335 x-0.7513$ & $y=-3.2336 x-0.751$ & $y=-2.2931 x-0.5155$ & $y=-0.948 x-0.1974$ & $y=-0.9925 x-0.0375$ \\
\hline \multicolumn{7}{|c|}{ Circumferential enlarged intima } \\
\hline & & $\Delta \mathrm{VWd}$ & $\Delta$ Rlu & $\Delta \mathrm{INTd}$ & $\triangle \mathrm{MEDd}$ & $\triangle \mathrm{ADVd}$ \\
\hline stage 1 & $(25-27)$ & $y=0.9555 x+16.208$ & $y=1.9555 x+16.207$ & $y=2.6479 x+30.55$ & $y=1.3403 x+12.251$ & $y=-3.0328 x-26.592$ \\
\hline \multirow[t]{3}{*}{ stage 2} & $(9-11)$ & $y=-0.393 x-1.6902$ & $y=0.607 x-1.6902$ & $y=0.3482 x-4.828$ & $y=-0.3746 x+1.0657$ & $y=-0.3666 x+2.0711$ \\
\hline & $(5-7)$ & $y=-0.4888 x+3.075$ & $y=0.5112 x+3.075$ & $y=0.5739 x-5.6071$ & $y=-1.6454 x+12.777$ & $y=0.5827 x-4.0956$ \\
\hline & $(19-21)$ & $y=-0.5123 x+4.8784$ & $y=0.4877 x+4.8834$ & $y=-0.2322 x+0.3748$ & $y=-0.6747 x+0.555$ & $y=0.3947 x+3.9515$ \\
\hline
\end{tabular}




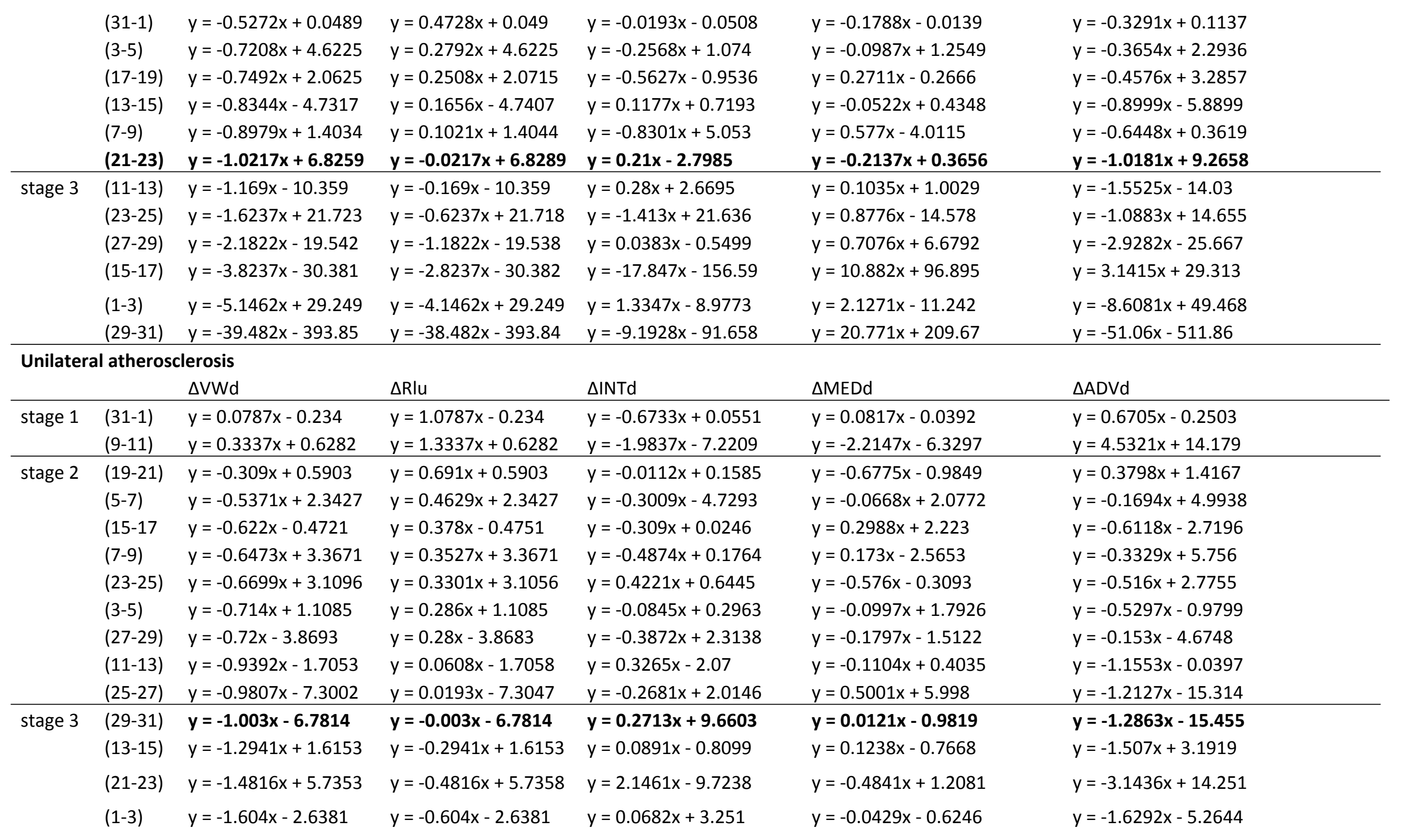


(17-19) $y=-2.8389 x-5.4709 \quad y=-1.8389 x-5.4709 \quad y=-3.7386 x-8.7361$

\begin{tabular}{|c|c|c|c|c|c|c|}
\hline \multicolumn{7}{|c|}{ Circumferential atherosclerosis } \\
\hline & & $\Delta \mathrm{VWd}$ & $\Delta$ Rlu & $\triangle \mathrm{INTd}$ & $\triangle \mathrm{MEDd}$ & $\triangle \mathrm{ADVd}$ \\
\hline \multirow[t]{3}{*}{ stage 1} & $(15-17)$ & $y=7.3116 x+52.816$ & $y=8.3116 x+52.816$ & $y=16.917 x+121.22$ & $y=-22.078 x-151.94$ & $y=12.472 x+83.535$ \\
\hline & $(7-9)$ & $y=0.4105 x-8.9468$ & $y=1.4105 x-8.9468$ & $y=-2.3582 x+25.243$ & $y=-1.1423 x+13.02$ & $y=3.9109 x-47.209$ \\
\hline & $(3-5)$ & $y=0.1175 x+7.0294$ & $y=1.1175 x+7.0294$ & $y=-1.4501 x-0.8144$ & $y=-0.8451 x-2.6804$ & $y=2.4127 x+10.524$ \\
\hline \multirow[t]{12}{*}{ stage 2} & $(11-13)$ & $y=-0.231 x+3.7454$ & $y=0.769 x+3.7444$ & $y=-1.03 x-16.602$ & $y=0.2261 x+4.864$ & $y=0.5728 x+15.487$ \\
\hline & $(27-29)$ & $y=-0.3663 x-3.8027$ & $y=0.6336 x-3.8311$ & $y=-0.6447 x-5.5001$ & $y=0.1906 x+1.7906$ & $y=0.0878 x-0.1002$ \\
\hline & $(25-27)$ & $y=-0.421 x-3.5213$ & $y=0.579 x-3.5213$ & $y=-0.4459 x-0.5729$ & $y=-0.0456 x+0.5292$ & $y=0.0705 x-3.4786$ \\
\hline & $(9-11)$ & $y=-0.5086 x-2.4846$ & $y=0.4914 x-2.4746$ & $y=-0.5706 x+0.9763$ & $y=0.0501 x-2.2035$ & $y=0.0119 x-1.2544$ \\
\hline & $(31-1)$ & $y=-0.5935 x+0.1002$ & $y=0.4065 x+0.0999$ & $y=-0.5473 x-0.0048$ & $y=-0.0343 x+0.0145$ & $y=-0.0343 x+0.0145$ \\
\hline & $(1-3)$ & $y=-0.6217 x+1.9193$ & $y=0.3783 x+1.9203$ & $y=-0.115 x-1.5121$ & $y=-0.7722 x-1.2299$ & $y=0.2655 x+4.6623$ \\
\hline & $(23-25)$ & $y=-0.6492 x+1.9002$ & $y=0.3508 x+1.9002$ & $y=-0.5531 x+8.8274$ & $y=0.0167 x-3.0843$ & $y=-0.1128 x-3.8509$ \\
\hline & $(13-15)$ & $y=-0.7407 x-7.7683$ & $y=0.2593 x-7.7683$ & $y=-1.4841 x-16.453$ & $y=0.9345 x+13.44$ & $y=-0.1911 x-4.7538$ \\
\hline & $(19-21)$ & $y=-0.8529 x+2.7078$ & $y=0.1471 x+2.7038$ & $y=-0.1555 x+2.0917$ & $y=-0.4562 x-3.0373$ & $y=-0.2412 x+3.6494$ \\
\hline & $(29-31)$ & $y=-0.8567 x-4.4847$ & $y=0.1433 x-4.4852$ & $y=-0.102 x+2.0679$ & $y=-1.0908 x-5.2542$ & $y=0.3362 x-1.2989$ \\
\hline & $(5-7)$ & $y=-0.9203 x+5.0949$ & $y=0.0797 x+5.0949$ & $y=-0.0077 x-0.5026$ & $y=-0.0369 x-0.2901$ & $y=-0.8757 x+5.8877$ \\
\hline & $(21-23)$ & $y=-0.9466 x+6.0568$ & $y=0.0534 x+6.0548$ & $y=-0.527 x+1.875$ & $y=-0.1509 x+0.3522$ & $y=-0.2687 x+3.8266$ \\
\hline stage 3 & $(17-19)$ & $y=-1.7118 x-9.6206$ & $y=-0.7118 x-9.6206$ & $y=0.6809 x+12$ & $y=0.581 x+4.5885$ & $y=0.581 x+4.5885$ \\
\hline \multicolumn{7}{|c|}{ Extreme unilateral atherosclerosis } \\
\hline & & $\Delta \mathrm{VWd}$ & $\Delta$ Rlu & $\Delta \mathrm{INTd}$ & $\triangle \mathrm{MEDd}$ & $\triangle \mathrm{ADVd}$ \\
\hline \multirow[t]{8}{*}{ stage 2} & $(5-7)$ & $y=-0.2939 x+4.9353$ & $y=0.7061 x+4.9353$ & $y=-0.3342 x+2.9221$ & $y=-0.1302 x+0.3114$ & $y=0.1706 x+1.7009$ \\
\hline & $(9-11)$ & $y=-0.3091 x-0.6248$ & $y=0.6909 x-0.6248$ & $y=-0.9362 x-18.244$ & $y=0.1113 x+4.4967$ & $y=0.5157 x+13.121$ \\
\hline & $(1-3)$ & $y=-0.4058 x+2.0641$ & $y=0.5942 x+2.0641$ & $y=-0.7378 x-0.6671$ & $y=0.0959 x-0.4716$ & $y=0.2361 x+3.2028$ \\
\hline & $(7-9)$ & $y=-0.5283 x-1.1638$ & $y=0.4717 x-1.1638$ & $y=-0.4966 x-0.5045$ & $y=-0.0094 x-0.1072$ & $y=-0.0223 x-0.555$ \\
\hline & $(15-17)$ & $y=-0.6198 x-2.9083$ & $y=0.3802 x-2.9083$ & $y=-0.735 x-9.6632$ & $y=0.3992 x+7.4305$ & $y=-0.2841 x-0.6776$ \\
\hline & $(11-13)$ & $y=-0.6462 x+1.8993$ & $y=0.3538 x+1.8993$ & $y=-1.8108 x-0.972$ & $y=1.1589 x+3.6811$ & $y=0.0057 x-0.8048$ \\
\hline & $(19-21)$ & $y=-0.7316 x-2.4254$ & $y=0.2684 x-2.4254$ & $y=38.703 x+735.98$ & $y=-17.382 x-324.65$ & $y=-22.051 x-413.7$ \\
\hline & $(3-5)$ & $y=-0.7372 x+9.0328$ & $y=0.2628 x+9.0328$ & $y=-0.7389 x+6.6779$ & $y=0.023 x+0.4632$ & $y=-0.0213 x+0.9907$ \\
\hline
\end{tabular}




\begin{tabular}{llllllll} 
& $(31-1)$ & $y=-0.8262 x+0.0652$ & $y=0.1738 x+0.0645$ & $y=0.1028 x+0.0109$ & $y=-0.4553 x-0.0136$ & $y=-0.4737 x+0.0678$ \\
& $(29-31)$ & $y=-\mathbf{0 . 8 3 9 5 x - 0 . 2 2 9}$ & $y=\mathbf{0 . 1 6 0 5 x - 0 . 2 2 9}$ & $y=-1.3479 x+8.1418$ & $y=\mathbf{0 . 3 2 3 2 x - 3 . 0 8 9 9}$ & $y=\mathbf{0 . 1 8 5 2 x - 5 . 2 7 7 8}$ \\
\hline stage 3 & $(23-25)$ & $y=-1.3628 x-6.5266$ & $y=-0.3628 x-6.5266$ & $y=-3.8562 x-25.599$ & $y=2.1118 x+18.85$ & $y=0.3816 x+0.2274$ \\
& $(27-29)$ & $y=-1.3843 x+4.4681$ & $y=-0.3843 x+4.4681$ & $y=-0.0526 x-15.13$ & $y=0.2781 x-0.3223$ & $y=-1.6099 x+19.921$ \\
& $(13-15)$ & $y=-1.8098 x-5.6616$ & $y=-0.8098 x-5.6616$ & $y=-0.0513 x+7.0871$ & $y=0.0246 x-5.7205$ & $y=-1.7831 x-7.0192$ \\
& $(21-23)$ & $y=-1.9352 x-10.524$ & $y=-0.9352 x-10.524$ & $y=-0.6001 x+0.9081$ & $y=-1.9618 x-19.55$ & $y=0.6267 x+8.1093$ \\
$(25-27)$ & $y=-2.1057 x-9.653$ & $y=-1.1057 x-9.653$ & $y=1.3281 x+13.742$ & $y=0.2048 x+1.3559$ & $y=-3.6386 x-24.749$ \\
$(17-19)$ & $y=-7.6744 x-201.48$ & $y=-6.6744 x-201.48$ & $y=-18.023 x-503.12$ & $y=8.7022 x+247.94$ & $y=1.6466 x+53.698$ \\
\hline
\end{tabular}

204

205

$\Delta$ Rlu-VWd was used as the reference. Triplets are arranged in descending order based on $\Delta$ Rlu-VWd trendline a-values. Bold marks the transition point from stage 2 to stage 3 by $\Delta$ Rlu a-value $\approx 0$ and $\Delta \mathrm{VWd}$ a-value $\approx 1$. 
The strictly ordered course of $\Delta \mathrm{VWd}$ and $\Delta \mathrm{Rlu}$ was not followed by a similar $\Delta \mathrm{INTd}$, triplets.

The same $\Delta \mathrm{VWd}$ trendline a-value in different sections regardless of pathology was

(trendline slope) but on a different level).

\section{Triplet course expressed by semi-ranges}

The paths followed in all sections and triplets expressed by semi-ranges (last value in triplet series minus first value) are presented in Table 6 . The systems order encountered in Table 5 and $\Delta$ Rlu semi-ranges $>0$, stage 2 by antagonistic semi-ranges, and both $<0$ in stage 3 . various sections. The changeover from stage 2 to stage 3 was recognizable by semi-range

222 value $\Delta \mathrm{Rlu} \approx 0$, accompanied by similar semi-ranges for $\Delta \mathrm{Rlu}-\mathrm{VWd}$ and $\Delta \mathrm{VWd}$ but greatly. 
Table 6. Semi-ranges Comprising the Last Value in a Triplet Series Minus the First Value.

Normal

\begin{tabular}{llcccccccc} 
& & $\Delta$ T-fu1 & $\Delta$ T-FU2 & $\Delta$ Rlu-VWd & $\Delta$ VWd & $\Delta$ Rlu & $\Delta I N T d$ & $\Delta$ MEDd & $\Delta$ ADVd \\
\hline stage 1 & $(11-13)$ & -9.5 & -2.8 & 0.5 & 3.8 & 4.3 & -1.8 & 4.4 & 1.2 \\
& $(3-5)$ & -9.2 & 4.0 & 3.7 & 3.5 & 7.2 & -1.1 & 4.2 & 0.3 \\
\hline stage 2 & $(19-21)$ & -7.5 & -18.8 & -8.6 & 1.7 & -6.9 & 0.2 & 3.1 & -1.6 \\
& $(23-25)$ & 4.2 & 10.6 & 4.8 & -1.0 & 3.7 & 0.5 & -1.7 & 0.2 \\
& $(13-15)$ & 1.3 & 2.4 & 1.1 & -0.2 & 0.8 & 0.2 & -0.5 & 0.0 \\
& $(9-11)$ & -9.0 & -6.3 & -2.8 & 0.7 & -2.0 & 1.6 & 3.3 & -4.1 \\
& $(27-29)$ & -0.5 & 8.4 & 3.4 & -1.6 & 1.8 & 2.5 & -0.4 & -3.7 \\
& $(21-23)$ & -7.6 & 23.7 & 9.1 & -5.4 & 3.7 & -5.1 & 0.7 & -1.0 \\
& $(17-19)$ & -6.1 & -23.4 & -9.0 & 5.4 & -3.7 & -0.8 & 3.8 & 2.3 \\
& $(7-9)$ & 2.7 & -21.7 & -8.2 & 5.2 & -3.0 & -0.1 & 0.8 & 4.5 \\
& $(1-3)$ & -35.7 & 35.2 & 13.3 & -8.6 & 4.8 & -5.0 & 9.0 & -12.6 \\
& $(31-1)$ & 0.1 & -1.6 & -0.6 & 0.4 & -0.1 & 0.0 & 0.1 & 0.3 \\
& $(29-31)$ & 10.6 & 13.7 & 4.7 & -4.3 & 0.4 & -3.5 & -5.0 & 4.1 \\
stage 3 & $(5-7)$ & 0.3 & -12.6 & -3.7 & 5.2 & 1.4 & -1.2 & 1.6 & 4.7 \\
& $(25-27)$ & 4.1 & -5.4 & -1.5 & 2.4 & 1.0 & 1.9 & -0.6 & 1.1 \\
& $(15-17)$ & 1.5 & -6.9 & -1.1 & 4.7 & 3.6 & 2.5 & 1.0 & 1.1 \\
\hline & sum & -60.1 & -1.4 & 5.2 & 11.8 & 17.0 & -9.0 & 24.0 & -3.2 \\
\hline
\end{tabular}

Circumferential enlarged intima

\begin{tabular}{cccccccccc} 
& & $\Delta$ T-fu1 & $\Delta$ T-FU2 & $\Delta$ Rlu-VWd & $\Delta$ VWd & $\Delta$ Rlu & $\Delta$ INTd & $\Delta$ MEDd & $\Delta$ ADVd \\
\hline stage 1 & $(25-27)$ & 14.4 & -4.9 & -4.7 & -4.5 & -9.2 & -12.4 & -6.3 & 14.2 \\
\hline stage 2 & $(9-11)$ & -11.9 & -39.1 & -16.3 & 6.4 & -9.9 & -5.7 & 6.1 & 6.0 \\
& $(5-7)$ & -24.9 & -13.9 & -5.6 & 2.7 & -2.9 & -3.2 & 9.2 & -3.3 \\
& $(19-21)$ & 24.8 & 41.3 & 16.4 & -8.4 & 8.0 & -3.8 & -11.1 & 6.5 \\
& $(31-1)$ & 0.0 & -2.5 & -1.0 & 0.5 & -0.5 & 0.0 & 0.2 & 0.3 \\
& $(3-5)$ & -6.5 & 41.9 & 15.4 & -11.1 & 4.3 & -4.0 & -1.5 & -5.6 \\
& $(17-19)$ & -28.0 & 49.3 & 17.9 & -13.4 & 4.5 & -10.1 & 4.9 & -8.2 \\
& $(13-15)$ & -16.6 & 69.4 & 24.5 & -20.4 & 4.1 & 2.9 & -1.3 & -22.0 \\
& $(7-9)$ & -64.4 & 70.9 & 24.5 & -22.0 & 2.5 & -20.3 & 14.1 & -15.8 \\
& $(21-23)$ & -12.3 & 97.3 & 32.2 & -32.9 & -0.7 & 6.8 & -6.9 & -32.8 \\
\hline stage 3 & $(11-13)$ & -17.7 & 37.9 & 12.0 & -14.0 & -2.0 & 3.3 & 1.2 & -18.6 \\
& $(23-25)$ & -30.9 & 26.3 & 7.3 & -11.8 & -4.5 & -10.2 & 6.4 & -7.9 \\
& $(27-29)$ & 25.0 & -24.2 & -5.8 & 12.7 & 6.9 & -0.2 & -4.1 & 17.0 \\
& $(15-17)$ & 29.9 & -4.8 & -0.8 & 3.1 & 2.3 & 14.6 & -8.9 & -2.6 \\
& $(1-3)$ & 10.3 & -6.4 & -0.9 & 4.6 & 3.7 & -1.2 & -1.9 & 7.7 \\
& $(29-31)$ & 16.1 & -6.5 & -0.2 & 6.2 & 6.1 & 1.4 & -3.3 & 8.1 \\
\hline & & & & - & & & & \\
& sum & -92.8 & 331.9 & 114.8 & 102.2 & 12.6 & -42.1 & -3.1 & -56.9 \\
\hline
\end{tabular}

Unilateral atherosclerosis

$\Delta$ T-fu1 $\quad \triangle$ T-FU2 $\quad \triangle$ Rlu-VWd $\triangle$ VWd $\triangle$ Rlu $\triangle$ INTd $\triangle$ MEDd $\triangle$ ADVd

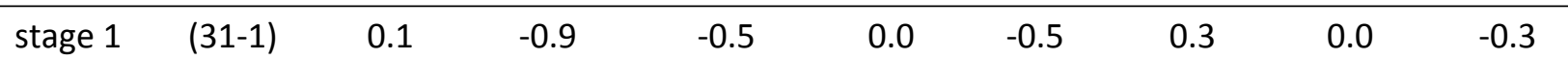




\begin{tabular}{cccccccccc} 
& $(9-11)$ & -11.4 & -2.7 & -1.6 & -0.5 & -2.2 & 3.2 & 3.6 & -7.4 \\
\hline stage 2 & $(19-21)$ & 11.7 & 15.6 & 6.8 & -2.1 & 4.7 & -0.1 & -4.6 & 2.6 \\
& $(5-7)$ & 5.1 & -38.2 & -15.1 & 8.1 & -7.0 & 4.5 & 1.0 & 2.6 \\
& $(15-17$ & -25.1 & 43.4 & 16.5 & -10.3 & 6.3 & -5.1 & 4.9 & -10.1 \\
& $(7-9)$ & -25.3 & 57.4 & 21.7 & -14.0 & 7.6 & -10.6 & 3.7 & -7.2 \\
& $(23-25)$ & -10.7 & -27.0 & -10.1 & 6.8 & -3.3 & -4.3 & 5.8 & 5.2 \\
& $(3-5)$ & -6.8 & 44.5 & 16.4 & -11.7 & 4.7 & -1.4 & -1.6 & -8.7 \\
& $(27-29)$ & 3.3 & -49.6 & -18.2 & 13.1 & -5.1 & 7.1 & 3.3 & 2.8 \\
& $(11-13)$ & -5.5 & 26.7 & 9.1 & -8.5 & 0.6 & 3.0 & -1.0 & -10.5 \\
& $(25-27)$ & 26.1 & -31.4 & -10.5 & 10.3 & -0.2 & 2.8 & -5.3 & 12.8 \\
\hline \multirow{2}{*}{ stage 3 } & $(29-31)$ & 6.7 & -19.3 & -6.4 & 6.4 & 0.0 & -1.7 & -0.1 & 8.3 \\
& $(13-15)$ & -14.8 & 29.2 & 8.9 & -11.5 & -2.6 & 0.8 & 1.1 & -13.4 \\
& $(21-23)$ & 0.1 & -7.9 & -2.3 & 3.4 & 1.1 & -4.9 & 1.1 & 7.2 \\
& $(1-3)$ & 4.1 & -10.0 & -2.8 & 4.5 & 1.7 & -0.2 & 0.1 & 4.5 \\
& $(17-19)$ & -6.2 & 3.0 & 0.6 & -1.8 & -1.1 & -2.3 & 1.5 & -0.9 \\
\hline & sum & -48.7 & 32.6 & 12.4 & -7.9 & 4.5 & -8.8 & 13.6 & -12.6 \\
\hline
\end{tabular}

Circumferential atherosclerosis

\begin{tabular}{cccccccccc} 
& & $\Delta$ FU1 & $\Delta$ T-FU2 & $\Delta$ Rlu-VWd & $\Delta$ VWd & $\Delta$ Rlu & $\Delta$ INTd & $\Delta$ MEDd & $\Delta$ ADVd \\
\hline stage 1 & $(15-17)$ & 55.6 & -4.0 & 0.8 & 5.5 & 6.3 & 12.8 & -16.7 & 9.4 \\
& $(7-9)$ & 7.5 & 3.1 & 2.0 & 0.8 & 2.8 & -4.6 & -2.2 & 7.7 \\
& $(3-5)$ & 12.8 & 9.1 & 4.8 & 0.6 & 5.4 & -7.0 & -4.1 & 11.7 \\
\hline stage 2 & $(11-13)$ & 17.7 & -43.3 & -19.4 & 4.5 & -14.9 & 20.0 & -4.4 & -11.1 \\
& $(27-29)$ & -43.3 & 109.3 & 46.2 & -16.9 & 29.3 & -29.8 & 8.8 & 4.1 \\
& $(25-27)$ & 16.2 & -138.4 & -57.2 & 24.1 & -33.1 & 25.5 & 2.6 & -4.0 \\
& $(9-11)$ & 89.1 & -339.3 & -135.3 & 68.8 & -66.5 & 77.2 & -6.8 & -1.6 \\
& $(31-1)$ & 0.6 & -3.1 & -1.2 & 0.7 & -0.5 & 0.6 & 0.0 & 0.0 \\
& $(1-3)$ & 14.3 & 22.0 & 8.4 & -5.2 & 3.2 & -1.0 & -6.5 & 2.2 \\
& $(23-25)$ & 47.6 & -180.3 & -68.1 & 44.2 & -23.9 & 37.7 & -1.1 & 7.7 \\
& $(13-15)$ & 48.7 & -37.7 & -13.8 & 10.2 & -3.6 & 20.4 & -12.9 & 2.6 \\
& $(19-21)$ & 13.3 & 73.7 & 25.8 & -22.0 & 3.8 & -4.0 & -11.8 & -6.2 \\
& $(29-31)$ & -10.7 & -12.6 & -4.4 & 3.8 & -0.6 & 0.5 & 4.8 & -1.5 \\
& $(5-7)$ & -15.6 & 56.3 & 19.3 & -17.7 & 1.5 & -0.1 & -0.7 & -16.9 \\
& $(21-23)$ & -13.9 & $\mathbf{8 3 . 1}$ & $\mathbf{2 8 . 2}$ & $-\mathbf{2 6 . 7}$ & $\mathbf{1 . 5}$ & $-\mathbf{- 1 4 . 9}$ & -4.3 & -7.6 \\
\hline stage 3 & $(17-19)$ & 16.8 & -18.0 & -4.9 & 8.3 & 3.5 & -3.3 & -2.8 & 14.4 \\
\hline & sum & 256.7 & -420.1 & -168.7 & 82.8 & -85.8 & 129.9 & -58.0 & 10.9 \\
\hline
\end{tabular}

Extreme unilateral atherosclerosis

\begin{tabular}{ccccccccc} 
& $\Delta \mathrm{T}-\mathrm{fu} 1$ & $\Delta \mathrm{T}-\mathrm{FU} 2$ & $\Delta \mathrm{Rlu}-\mathrm{VWd}$ & $\Delta \mathrm{VWd}$ & $\Delta \mathrm{Rlu}$ & $\Delta \mathrm{INTd}$ & $\Delta \mathrm{MEDd}$ & $\Delta \mathrm{ADVd}$ \\
\hline$(5-7)$ & 3.6 & 85.4 & 37.2 & -10.9 & 26.3 & -12.4 & -4.8 & 6.4 \\
$(9-11)$ & -26.4 & 94.8 & 41.1 & -12.7 & 28.4 & -38.4 & 4.6 & 21.2 \\
$(1-3)$ & -7.2 & 29.6 & 12.3 & -5.0 & 7.3 & -9.1 & 1.2 & 2.9 \\
$(7-9)$ & 108.2 & -547.2 & -216.4 & 114.3 & -102.1 & 107.5 & 2.0 & 4.8 \\
$(15-17)$ & 65.2 & -94.0 & -35.9 & 22.2 & -13.6 & 26.4 & -14.3 & 10.2 \\
$(11-13)$ & 93.2 & -59.8 & -22.6 & 14.6 & -8.0 & 40.9 & -26.2 & -0.1 \\
$(19-21)$ & 49.0 & 2.6 & 1.0 & -0.7 & 0.3 & 36.9 & -16.6 & -21.0 \\
$(3-5)$ & 28.8 & -97.9 & -35.8 & 26.4 & -9.4 & 26.4 & -0.8 & 0.8
\end{tabular}




\begin{tabular}{ccccccccc}
$(31-1)$ & 0.7 & 3.8 & 1.3 & -1.1 & 0.2 & 0.1 & -0.6 & -0.6 \\
$(29-31)$ & 16.5 & -25.9 & -9.1 & 7.7 & -1.5 & 12.3 & -3.0 & -1.7 \\
$(\mathbf{2 3 - 2 5 )}$ & -30.4 & 13.3 & 3.9 & -5.4 & -1.4 & -15.2 & $\mathbf{8 . 3}$ & 1.5 \\
$(27-29)$ & 29.0 & -44.3 & -13.1 & 18.1 & 5.0 & 0.7 & -3.6 & 21.1 \\
$(13-15)$ & -28.7 & 58.0 & 15.2 & -27.6 & -12.3 & -0.8 & 0.4 & -27.2 \\
$(21-23)$ & 38.5 & -39.7 & -10.1 & 19.5 & 9.4 & 6.0 & -6.3 & 19.8 \\
$(25-27)$ & 10.3 & -15.5 & -3.8 & 8.0 & 4.2 & -5.0 & -0.8 & 13.8 \\
$(17-19)$ & 39.4 & -11.3 & -1.2 & 9.0 & 7.8 & 21.0 & -10.2 & -1.9 \\
sum & 389.80 & -648.08 & -235.9 & 176.4 & -59.5 & 197.31 & -70.7 & 49.8 \\
\hline
\end{tabular}
transition point from stage 2 to stage 3.

The $\triangle \mathrm{INTd}, \triangle \mathrm{MEDd}$, and $\triangle \mathrm{ADVd}$ semi-ranges mutually changed from one triplet to the next, increase or decrease of one opposed to the decrease or increase of the other two, a counterbalancing system found in all sections regardless of histological rating. The summed values presented their mutual distribution: 'normal', semi-range $\triangle \mathrm{MEDd}$ opposed by semi-ranges $\triangle \mathrm{INTd}$ and $\triangle \mathrm{ADVd}$; 'circumferential enlarged intima', all three had the same sign; 'unilateral atherosclerosis', semi-range $\triangle \mathrm{MEDd}$ opposed by semi-ranges $\triangle \mathrm{INTd}$ and $\triangle \mathrm{ADVd}$; and 'circumferential atherosclerosis', semi-ranges $\triangle \mathrm{INTd}$ and $\triangle \mathrm{ADVd}$ opposed by $\triangle \mathrm{MEDd}$ (the same in 'extreme unilateral atherosclerosis'). The last two sections had the largest semi-range $\Delta \mathrm{INTd}$ increase together with semi-range $\triangle \mathrm{ADVd}$ growth opposed by semi-range $\triangle \mathrm{MEDd}$.

\section{Influence of $\Delta V W$ composition on $\Delta R l u$}

240 The individual relationship between $\triangle \mathrm{INTd}, \triangle \mathrm{MEDd}$, and $\triangle \mathrm{ADVd}$ and $\triangle \mathrm{Rlu}$ was not

241 elucidated. Stage 2 differed strongly from stage 1 and stage 3, most obviously by summed

242 values $\Delta \mathrm{VWd}$ in 'normal', 'circumferential enlarged intima', and 'unilateral atherosclerosis'

243 sections being predominantly negative due to $-\triangle \mathrm{INT},-\triangle \mathrm{ADVd}$, and $+\Delta \mathrm{MEDd}$. In contrast, 'circumferential atherosclerosis' sections presented predominantly positive $\Delta \mathrm{VWd}$ values due 
to $+\triangle \mathrm{INTd},-\triangle \mathrm{MEDd}$, and $-\triangle \mathrm{ADVd}$, whereas 'extreme unilateral atherosclerosis' had positive

$246 \quad \Delta \mathrm{VWd}$ values due to $+\Delta \mathrm{INTd},-\Delta \mathrm{MEDd}$, and $+\Delta \mathrm{ADVd}$

247 Comparisons of semi-range ratios $\Delta \mathrm{INT}, \Delta \mathrm{MEDd}$, or $\triangle \mathrm{ADVd}$ to $\Delta \mathrm{VWd}$ and $\Delta \mathrm{Rlu}$ showed

248 variability in the single semi-range values (Table 7). Naturally, the summed semi-range

249 values $\triangle \mathrm{INTd}, \triangle \mathrm{MEDd}$, and $\triangle \mathrm{ADVd}$ in proportion to semi-range $\Delta \mathrm{VWd}$ equaled 1 , but in

250 proportion to semi-range $\Delta$ Rlu a systemic course developed similar to the semi-range ratio of

$251 \Delta$ VWd to $\Delta$ Rlu. These results were found in all sections independent of semi-range $\Delta \mathrm{INTd}$,

$252 \Delta \mathrm{MEDd}$, or $\triangle \mathrm{ADVd}$ values. 


\section{Normal}

\begin{tabular}{|c|c|c|c|c|c|c|c|c|c|c|}
\hline & & $\Delta \mathrm{INTd} / \mathrm{L}$ & $\mathrm{IEDd} / \Delta \mathrm{AL}$ & dd ratio $\Delta$ & & $\Delta \mathrm{INTd} / \Delta$ & $1 \mathrm{EDd} / \Delta \mathrm{Al}$ & $/ \mathrm{d}$ ratio $\Delta$ & & \\
\hline & & $\Delta \mathrm{INTd}$ & $\triangle \mathrm{MEDd}$ & $\triangle \mathrm{ADVd}$ & sum & $\Delta \mathrm{INTd}$ & $\triangle \mathrm{MEDd}$ & $\triangle \mathrm{ADVd}$ & sum & $\Delta \mathrm{VWd} / \Delta \mathrm{Rlu}$ \\
\hline stage 1 & $(11-13)$ & -0.5 & 1.2 & 0.3 & 1.0 & -0.4 & 1.0 & 0.3 & 0.88 & 0.88 \\
\hline & $(3-5)$ & -0.3 & 1.2 & 0.1 & 1.0 & -0.1 & 0.6 & 0.0 & 0.48 & 0.48 \\
\hline stage 2 & $(19-21)$ & 0.1 & 1.8 & -0.9 & 1.0 & 0.0 & -0.4 & 0.2 & -0.25 & -0.25 \\
\hline & $(23-25)$ & -0.5 & 1.7 & -0.2 & 1.0 & 0.1 & -0.5 & 0.1 & -0.27 & -0.27 \\
\hline & $(13-15)$ & -1.0 & 2.2 & -0.2 & 1.0 & 0.3 & -0.6 & 0.1 & -0.27 & -0.27 \\
\hline & $(9-11)$ & 2.2 & 4.5 & -5.7 & 1.0 & -0.8 & -1.6 & 2.0 & -0.36 & -0.36 \\
\hline & $(27-29)$ & -1.6 & 0.2 & 2.3 & 1.0 & 1.4 & -0.2 & -2.0 & -0.87 & -0.87 \\
\hline & $(21-23)$ & 0.9 & -0.1 & 0.2 & 1.0 & -1.4 & 0.2 & -0.3 & -1.45 & -1.45 \\
\hline & $(17-19)$ & -0.1 & 0.7 & 0.4 & 1.0 & 0.2 & -1.0 & -0.6 & -1.47 & -1.47 \\
\hline & $(7-9)$ & 0.0 & 0.2 & 0.9 & 1.0 & 0.0 & -0.3 & -1.5 & -1.75 & -1.75 \\
\hline & $(1-3)$ & 0.6 & -1.1 & 1.5 & 1.0 & -1.1 & 1.9 & -2.6 & -1.80 & -1.80 \\
\hline & $(31-1)$ & 0.1 & 0.3 & 0.7 & 1.0 & -0.2 & -0.8 & -2.0 & -2.96 & -2.96 \\
\hline & $(29-31)$ & 0.8 & 1.2 & -1.0 & 1.0 & -9.6 & -13.7 & 11.4 & -11.90 & -11.90 \\
\hline stage 3 & $(5-7)$ & -0.2 & 0.3 & 0.9 & 1.0 & -0.8 & 1.1 & 3.3 & 3.59 & 3.59 \\
\hline & $(25-27)$ & 0.8 & -0.2 & 0.4 & 1.0 & 2.0 & -0.6 & 1.1 & 2.53 & 2.53 \\
\hline & $(15-17)$ & 0.5 & 0.2 & 0.2 & 1.0 & 0.7 & 0.3 & 0.3 & 1.31 & 1.31 \\
\hline
\end{tabular}

\section{Circumferential enlarged intima}

\begin{tabular}{|c|c|c|c|c|c|c|c|c|c|c|}
\hline & & \multicolumn{4}{|c|}{$\Delta \mathrm{INTd} / \Delta \mathrm{MEDd} / \Delta \mathrm{ADVd}$ ratio $\Delta \mathrm{VWd}$} & \multicolumn{4}{|c|}{$\Delta \mathrm{INTd} / \Delta \mathrm{MEDd} / \triangle \mathrm{ADV}$ d ratio $\Delta \mathrm{Rlu}$} & \multirow[b]{2}{*}{$\Delta \mathrm{VWd} / \Delta \mathrm{Rlu}$} \\
\hline & & $\Delta \mathrm{INTd}$ & $\triangle \mathrm{MEDd}$ & $\triangle \mathrm{ADVd}$ & sum & $\Delta \mathrm{INTd}$ & $\triangle \mathrm{MEDd}$ & $\triangle \mathrm{ADVd}$ & sum & \\
\hline stage 1 & $(25-27)$ & 2.8 & 1.4 & -3.2 & 1.0 & 1.4 & 0.7 & -1.6 & 0.5 & 0.5 \\
\hline \multirow[t]{2}{*}{ stage 2} & $(9-11)$ & -0.9 & 1.0 & 0.9 & 1.0 & 0.6 & -0.6 & -0.6 & -0.6 & -0.6 \\
\hline & $(5-7)$ & -1.2 & 3.4 & -1.2 & 1.0 & 1.1 & -3.2 & 1.1 & -1.0 & -1.0 \\
\hline
\end{tabular}




\begin{tabular}{cccccc|cccc|c} 
& $(19-21)$ & 0.5 & 1.3 & -0.8 & 1.0 & -0.5 & -1.4 & 0.8 & -1.1 & -1.1 \\
& $(31-1)$ & 0.0 & 0.3 & 0.6 & 1.0 & 0.0 & -0.4 & -0.7 & -1.1 & -1.1 \\
& $(3-5)$ & 0.4 & 0.1 & 0.5 & 1.0 & -0.9 & -0.4 & -1.3 & -2.6 & -2.6 \\
& $(17-19)$ & 0.8 & -0.4 & 0.6 & 1.0 & -2.2 & 1.1 & -1.8 & -3.0 & -3.0 \\
& $(13-15)$ & -0.1 & 0.1 & 1.1 & 1.0 & 0.7 & -0.3 & -5.4 & -5.0 & -5.0 \\
& $(7-9)$ & 0.9 & -0.6 & 0.7 & 1.0 & -8.1 & 5.6 & -6.3 & -8.8 & -8.8 \\
& $(21-23)$ & -0.2 & $\mathbf{0 . 2}$ & $\mathbf{1 . 0}$ & $\mathbf{1 . 0}$ & -9.7 & $\mathbf{9 . 8}$ & $\mathbf{4 6 . 9}$ & $\mathbf{4 7 . 1}$ & $\mathbf{4 7 . 1}$ \\
\hline stage 3 & $(11-13)$ & -0.2 & -0.1 & 1.3 & 1.0 & -1.7 & -0.6 & 9.2 & 6.9 & 6.9 \\
& $(23-25)$ & 0.9 & -0.5 & 0.7 & 1.0 & 2.3 & -1.4 & 1.7 & 2.6 & 2.6 \\
& $(27-29)$ & 0.0 & -0.3 & 1.3 & 1.0 & 0.0 & -0.6 & 2.5 & 1.8 & 1.8 \\
& $(15-17)$ & 4.7 & -2.8 & -0.8 & 1.0 & 6.3 & -3.9 & -1.1 & 1.4 & 1.4 \\
& $(1-3)$ & -0.3 & -0.4 & 1.7 & 1.0 & -0.3 & -0.5 & 2.1 & 1.2 & 1.2 \\
& $(29-31)$ & 0.2 & -0.5 & 1.3 & 1.0 & 0.2 & -0.5 & 1.3 & 1.0 & 1.0 \\
\hline
\end{tabular}

\begin{tabular}{|c|c|c|c|c|c|c|c|c|c|c|}
\hline \multicolumn{11}{|c|}{ Unilateral atherosclerosis } \\
\hline & & \multicolumn{4}{|c|}{$\Delta \mathrm{INTd} / \Delta \mathrm{MEDd} / \Delta \mathrm{ADVd}$ ratio $\Delta \mathrm{VWd}$} & \multicolumn{4}{|c|}{$\Delta \mathrm{INTd} / \triangle \mathrm{MEDd} / \triangle \mathrm{ADV}$ d ratio $\Delta \mathrm{Rlu}$} & \multirow[b]{2}{*}{$\Delta \mathrm{VWd} / \Delta \mathrm{Rlu}$} \\
\hline & & $\Delta \mathrm{INTd}$ & $\triangle \mathrm{MEDd}$ & $\triangle \mathrm{ADVd}$ & som & $\Delta \mathrm{INTd}$ & $\triangle \mathrm{MEDd}$ & $\triangle \mathrm{ADVd}$ & som & \\
\hline \multirow{2}{*}{ stage 1} & $(31-1)$ & -8.6 & 1.0 & 8.5 & 1.0 & -0.6 & 0.1 & 0.6 & 0.07 & 0.07 \\
\hline & $(9-11)$ & -5.9 & -6.6 & 13.6 & 1.0 & -1.5 & -1.7 & 3.4 & 0.25 & 0.25 \\
\hline \multirow[t]{9}{*}{ stage 2} & $(19-21)$ & 0.0 & 2.2 & -1.2 & 1.0 & 0.0 & -1.0 & 0.5 & -0.45 & -0.45 \\
\hline & $(5-7)$ & 0.6 & 0.1 & 0.3 & 1.0 & -0.6 & -0.1 & -0.4 & -1.16 & -1.16 \\
\hline & $(15-17$ & 0.5 & -0.5 & 1.0 & 1.0 & -0.8 & 0.8 & -1.6 & -1.65 & -1.65 \\
\hline & $(7-9)$ & 0.8 & -0.3 & 0.5 & 1.0 & -1.4 & 0.5 & -0.9 & -1.84 & -1.84 \\
\hline & $(23-25)$ & -0.6 & 0.9 & 0.8 & 1.0 & 1.3 & -1.7 & -1.6 & -2.03 & -2.03 \\
\hline & (3-5) & 0.1 & 0.1 & 0.7 & 1.0 & -0.3 & -0.3 & -1.9 & -2.50 & -2.50 \\
\hline & $(27-29)$ & 0.5 & 0.2 & 0.2 & 1.0 & -1.4 & -0.6 & -0.5 & -2.57 & -2.57 \\
\hline & $(11-13)$ & -0.3 & 0.1 & 1.2 & 1.0 & 5.4 & -1.8 & -19.0 & -15.44 & -15.44 \\
\hline & $(25-27)$ & 0.3 & -0.5 & 1.2 & 1.0 & -13.9 & 25.9 & -62.8 & -50.77 & -50.77 \\
\hline \multirow[t]{2}{*}{ stage 3} & $(29-31)$ & -0.3 & 0.0 & 1.3 & 1.0 & -91.8 & -4.1 & 435.3 & 339.40 & 339.40 \\
\hline & $(13-15)$ & -0.1 & -0.1 & 1.2 & 1.0 & -0.3 & -0.4 & 5.1 & 4.40 & 4.40 \\
\hline
\end{tabular}




\begin{tabular}{ccccc|cccc|c}
$(21-23)$ & -1.4 & 0.3 & 2.1 & 1.0 & -4.5 & 1.0 & 6.5 & 3.08 & 3.08 \\
$(1-3)$ & 0.0 & 0.0 & 1.0 & 1.0 & -0.1 & 0.1 & 2.7 & 2.66 & 2.66 \\
$(17-19)$ & 1.3 & -0.8 & 0.5 & 1.0 & 2.0 & -1.3 & 0.8 & 1.54 & 1.54 \\
\hline
\end{tabular}

\begin{tabular}{|c|c|c|c|c|c|c|c|c|c|c|}
\hline \multicolumn{11}{|c|}{ Circumferential atherosclerosis } \\
\hline & & \multicolumn{4}{|c|}{$\Delta \mathrm{INTd} / \Delta \mathrm{MEDd} / \Delta \mathrm{ADVd}$ ratio $\Delta \mathrm{VWd}$} & \multicolumn{4}{|c|}{$\Delta \mathrm{INTd} / \Delta \mathrm{MEDd} / \triangle \mathrm{ADVd}$ ratio $\Delta \mathrm{Rlu}$} & \multirow[b]{2}{*}{$\Delta \mathrm{VWd} / \Delta \mathrm{Rlu}$} \\
\hline & & $\Delta \mathrm{INTd}$ & $\triangle \mathrm{MEDd}$ & $\triangle \mathrm{ADVd}$ & sum & $\triangle \mathrm{INTd}$ & $\triangle \mathrm{MEDd}$ & $\triangle \mathrm{ADVd}$ & sum & \\
\hline \multirow[t]{3}{*}{ stage 1} & $(15-17)$ & 2.3 & -3.0 & 1.7 & 1.0 & 2.0 & -2.7 & 1.5 & 0.88 & 0.88 \\
\hline & (7-9) & -5.7 & -2.8 & 9.5 & 1.0 & -1.7 & -0.8 & 2.8 & 0.29 & 0.29 \\
\hline & $(3-5)$ & -12.3 & -7.2 & 20.5 & 1.0 & -1.3 & -0.8 & 2.2 & 0.11 & 0.11 \\
\hline \multirow[t]{12}{*}{ stage 2} & $(11-13)$ & 4.5 & -1.0 & -2.5 & 1.0 & -1.3 & 0.3 & 0.7 & -0.30 & -0.30 \\
\hline & $(27-29)$ & 1.8 & -0.5 & -0.2 & 1.0 & -1.0 & 0.3 & 0.1 & -0.58 & -0.58 \\
\hline & $(25-27)$ & 1.1 & 0.1 & -0.2 & 1.0 & -0.8 & -0.1 & 0.1 & -0.73 & -0.73 \\
\hline & $(9-11)$ & 1.1 & -0.1 & 0.0 & 1.0 & -1.2 & 0.1 & 0.0 & -1.04 & -1.04 \\
\hline & $(31-1)$ & 0.9 & 0.1 & 0.0 & 1.0 & -1.3 & -0.1 & 0.0 & -1.46 & -1.46 \\
\hline & $(1-3)$ & 0.2 & 1.2 & -0.4 & 1.0 & -0.3 & -2.0 & 0.7 & -1.64 & -1.64 \\
\hline & $(23-25)$ & 0.9 & 0.0 & 0.2 & 1.0 & -1.6 & 0.0 & -0.3 & -1.85 & -1.85 \\
\hline & $(13-15)$ & 2.0 & -1.3 & 0.3 & 1.0 & -5.7 & 3.6 & -0.7 & -2.86 & -2.86 \\
\hline & $(19-21)$ & 0.2 & 0.5 & 0.3 & 1.0 & -1.1 & -3.1 & -1.6 & -5.80 & -5.80 \\
\hline & $(29-31)$ & 0.1 & 1.3 & -0.4 & 1.0 & -0.7 & -7.6 & 2.3 & -5.98 & -5.98 \\
\hline & (5-7) & 0.0 & 0.0 & 1.0 & 1.0 & -0.1 & -0.5 & -11.0 & -11.55 & -11.55 \\
\hline & $(21-23)$ & 0.6 & 0.2 & 0.3 & 1.0 & -9.9 & -2.8 & -5.0 & -17.74 & -17.74 \\
\hline stage 3 & $(17-19)$ & -0.4 & -0.3 & 1.7 & 1.0 & -1.0 & -0.8 & 4.2 & 2.40 & 2.40 \\
\hline
\end{tabular}

\begin{tabular}{|c|c|c|c|c|c|c|c|c|c|c|}
\hline \multicolumn{10}{|c|}{ Extreme unilateral atherosclerosis } & \multirow[b]{3}{*}{$\Delta \mathrm{VWd} / \Delta \mathrm{Rlu}$} \\
\hline & & \multicolumn{4}{|c|}{$\Delta \mathrm{INTd} / \Delta \mathrm{MEDd} / \triangle \mathrm{ADVd}$ ratio $\Delta \mathrm{VWd}$} & \multicolumn{4}{|c|}{$\Delta \mathrm{INTd} / \Delta \mathrm{MEDd} / \triangle \mathrm{ADV}$ d ratio $\Delta \mathrm{Rlu}$} & \\
\hline & & $\Delta \mathrm{INTd}$ & $\triangle \mathrm{MEDd}$ & $\triangle \mathrm{ADVd}$ & sum & $\Delta \mathrm{INTd}$ & $\triangle \mathrm{MEDd}$ & $\triangle \mathrm{ADVd}$ & sum & \\
\hline \multirow[t]{4}{*}{ stage 2} & $(5-7)$ & 1.1 & 0.4 & -0.6 & 1.0 & -0.5 & -0.2 & 0.2 & -0.42 & -0.42 \\
\hline & $(9-11)$ & 3.0 & -0.4 & -1.7 & 1.0 & -1.4 & 0.2 & 0.7 & -0.45 & -0.45 \\
\hline & $(1-3)$ & 1.8 & -0.2 & -0.6 & 1.0 & -1.2 & 0.2 & 0.4 & -0.68 & -0.68 \\
\hline & (7-9) & 0.9 & 0.0 & 0.0 & 1.0 & -1.1 & 0.0 & 0.0 & -1.12 & -1.12 \\
\hline
\end{tabular}




\begin{tabular}{llllll|cccc|c} 
& $(15-17)$ & 1.2 & -0.6 & 0.5 & 1.0 & -1.9 & 1.1 & -0.7 & -1.63 & -1.63 \\
& $(11-13)$ & 2.8 & -1.8 & 0.0 & 1.0 & -5.1 & 3.3 & 0.0 & -1.83 & -1.83 \\
& $(19-21)$ & -52.9 & 23.8 & 30.1 & 1.0 & 144.2 & -64.8 & -82.1 & -2.72 & -2.73 \\
& $(3-5)$ & 1.0 & 0.0 & 0.0 & 1.0 & -2.8 & 0.1 & -0.1 & -2.81 & -2.81 \\
& $(31-1)$ & -0.1 & 0.6 & 0.6 & 1.0 & 0.6 & -2.6 & -2.7 & -4.75 & -4.75 \\
& $(29-31)$ & 1.6 & -0.4 & -0.2 & 1.0 & -8.4 & 2.0 & 1.2 & -5.23 & -5.23 \\
& $(23-25)$ & $\mathbf{2 . 8}$ & -1.5 & -0.3 & 1.0 & 10.6 & -5.8 & -1.1 & $\mathbf{3 . 7 6}$ & 3.76 \\
\hline stage 3 & $(27-29)$ & 0.0 & -0.2 & 1.2 & 1.0 & 0.1 & -0.7 & 4.2 & 3.60 & 3.60 \\
& $(13-15)$ & 0.0 & 0.0 & 1.0 & 1.0 & 0.1 & 0.0 & 2.2 & 2.23 & 2.23 \\
& $(21-23)$ & 0.3 & -0.3 & 1.0 & 1.0 & 0.6 & -0.7 & 2.1 & 2.07 & 2.07 \\
& $(25-27)$ & -0.6 & -0.1 & 1.7 & 1.0 & -1.2 & -0.2 & 3.3 & 1.90 & 1.90 \\
& $(17-19)$ & 2.3 & -1.1 & -0.2 & 1.0 & 2.7 & -1.3 & -0.2 & 1.15 & 1.15
\end{tabular}

254 Their summed values in proportion to $\Delta \mathrm{VWd}$ and $\Delta \mathrm{Rlu}$. Triplets are arranged in descending order based on $\Delta \mathrm{Rlu}-\mathrm{VWd}$ trendline a-values. Bold marks the

transition from stage 2 to stage 3. 
Despite semi-range $\Delta \mathrm{INTd}, \Delta \mathrm{MEDd}$, and $\triangle \mathrm{ADVd}$ mutually variable interactions, semi-range

258 finding emphasizes that semi-ranges reliably reproduce measurement data.

259 Table 8. Trendline Formula $\Delta$ Rlu vs. $\Delta$ VWd Triplets.

\section{Normal}

\begin{tabular}{llll}
\multicolumn{3}{c}{$\Delta$ VWd } \\
\hline stage 1 & $(11-13)$ & $-2.9 \_0.9$ & $\mathrm{y}=1.1313 \mathrm{x}+3.4377$ \\
& $(3-5)$ & $-1.3 \_2.2$ & $\mathrm{y}=2.0784 \mathrm{x}-3.2453$ \\
\hline stage 2 & $(19-21)$ & $-0.3 \_1.4$ & $\mathrm{y}=-4.0422 \mathrm{x}+1.5327$ \\
& $(23-25)$ & $1.4 \_0.4$ & $\mathrm{y}=-3.6406 \mathrm{x}+1.8347$ \\
& $(13-15)$ & $-0.1 \_-0.3$ & $\mathrm{y}=-3.6478 \mathrm{x}-0.7137$ \\
& $(9-11)$ & $0.1 \_0.9$ & $\mathrm{y}=-2.8024 \mathrm{x}+2.5118$ \\
& $(27-29)$ & $0.3 \_-1.3$ & $\mathrm{y}=-1.154 \mathrm{x}+0.2855$ \\
& $(21-23)$ & $2.9 \_-2.5$ & $\mathrm{y}=-0.6874 \mathrm{x}-1.0597$ \\
& $(17-19)$ & $-2.9 \_2.4$ & $\mathrm{y}=-0.6823 \mathrm{x}-0.3664$ \\
& $(7-9)$ & $-3.9 \_1.3$ & $\mathrm{y}=-0.5714 \mathrm{x}+0.1617$ \\
& $(1-3)$ & $5.3 \_3.2$ & $\mathrm{y}=-0.5557 \mathrm{x}-0.6603$ \\
& $(31-1)$ & $3.5 \_3.9$ & $\mathrm{y}=-0.3379 \mathrm{x}-0.0314$ \\
& $(29-31)$ & $2.2 \_-2.1$ & $\mathrm{y}=-0.0841 \mathrm{x}+2.5138$ \\
\hline stage 3 & $(5-7)$ & -1.4 3.8 & $\mathrm{y}=0.2787 \mathrm{x}-0.7202$ \\
& $(25-27)$ & $-2.9 \_-0.5$ & $\mathrm{y}=0.3951 \mathrm{x}-0.2123$ \\
& $(15-17)$ & $-1.7 \_3.0$ & $\mathrm{y}=0.7638 \mathrm{x}-0.1772$ \\
\hline
\end{tabular}

Unilateral atherosclerosis

\begin{tabular}{|c|c|c|c|}
\hline & & VWd & \\
\hline \multirow[t]{2}{*}{ stage 1} & $(31-1)$ & $-0.49-0.53$ & $y=13.702 x+2.9726$ \\
\hline & $(9-11)$ & $0 \_-0.6$ & $y=3.9965 x-1.8825$ \\
\hline \multirow[t]{9}{*}{ stage 2} & $(19-21)$ & $2.2 \_0.1$ & $y=-2.2364 x+1.9104$ \\
\hline & $(5-7)$ & $-16.1-8$ & $y=-0.862 x+4.3621$ \\
\hline & $(15-17$ & $7.8-2.5$ & $y=-0.6078 x-0.762$ \\
\hline & $(7-9)$ & $2-12$ & $y=-0.5448 x+5.2015$ \\
\hline & $(23-25)$ & $0.5 \_7.3$ & $y=-0.4929 x+4.6382$ \\
\hline & $(3-5)$ & 5.7_-6 & $y=-0.4005 x+1.5525$ \\
\hline & $(27-29)$ & $-7.7 \_5.4$ & $y=-0.3889 x-5.3733$ \\
\hline & $(11-13)$ & $0.6 \_-7.9$ & $y=-0.0648 x-1.8162$ \\
\hline & $(25-27)$ & $-1.7 \_8.6$ & $y=-0.0197 x-7.4485$ \\
\hline \multirow[t]{4}{*}{ stage 3} & $(29-31)$ & 3_9.5 & $y=0.0029 x-6.7615$ \\
\hline & $(13-15)$ & $3.7-7.7$ & $y=-0.6042 x+4.3451$ \\
\hline & $(21-23)$ & $-2.3 \_1.1$ & $y=0.3251 x+3.8714$ \\
\hline & $(1-3)$ & 1 1_5.5 & $y=0.3765 x-1.6447$ \\
\hline
\end{tabular}


(17-19) 2_0.2

$y=0.2272 x+1.2483$

\begin{tabular}{|c|c|c|c|}
\hline \multicolumn{4}{|c|}{ Circumferential enlarged intima } \\
\hline \multicolumn{4}{|c|}{$\Delta \mathrm{VWd}$} \\
\hline stage 1 & $(25-27)$ & 8.4_3.9 & $y=2.0466 x-16.965$ \\
\hline \multirow[t]{9}{*}{ stage 2} & $(9-11)$ & $-7 \_-0.6$ & $y=-1.5447 x-4.3011$ \\
\hline & $(5-7)$ & $-2.1 \_0.6$ & $y=-1.0458 x+6.2907$ \\
\hline & $(19-21)$ & 9.6_1.2 & $y=-0.9521 x+9.5283$ \\
\hline & $(31-1)$ & $4 \_4.5$ & $y=-0.897 x+0.0929$ \\
\hline & $(3-5)$ & $6.6-4.5$ & $y=-0.3873 x+6.413$ \\
\hline & $(17-19)$ & $-0.2 \_4.3$ & $y=-2.2438 x+3.6943$ \\
\hline & $(13-15)$ & $10.6-9.8$ & $y=-0.1984 x-5.6796$ \\
\hline & $(7-9)$ & 8.1_-13.9 & $y=-0.1138 x+1.5641$ \\
\hline & $(21-23)$ & $11 \_-22$ & $y=0.0212 x+6.6839$ \\
\hline \multirow[t]{6}{*}{ stage 3} & $(11-13)$ & 6.6_-7.4 & $y=0.1446 x-8.8613$ \\
\hline & $(23-25)$ & $2.6-14.4$ & $y=0.3841 x+13.374$ \\
\hline & $(27-29)$ & $-6.2 \_6.4$ & $y=0.5418 x-8.9512$ \\
\hline & $(15-17)$ & 3_6.1 & $y=0.7385 x-7.9461$ \\
\hline & $(1-3)$ & $-3.5 \_1.2$ & $y=0.8057 x+5.6836$ \\
\hline & $(29-31)$ & $-1.8 \_4.5$ & $y=0.9747 x-9.9709$ \\
\hline
\end{tabular}

Circumferential atherosclerosis

\begin{tabular}{llll}
\multicolumn{3}{c}{$\Delta$ VWd } \\
\hline stage 1 & $(15-17)$ & $-06 \_5.0$ & $y=1.1368 x-7.2236$ \\
& $(7-9)$ & $-4.5 \_-3.7$ & $y=3.4363 x+21.797$ \\
& $(3-5)$ & $6.4 \_6.9$ & $y=9.5084 x-59.809$ \\
\hline stage 2 & $(11-13)$ & $6.0 \_11.4$ & $y=-3.3284 x+16.211$ \\
& $(27-29)$ & $8.7 \_-8.3$ & $y=-1.7298 x-10.409$ \\
& $(25-27)$ & $-23 \_1.1$ & $y=-1.3753 x-8.3641$ \\
& $(9-11)$ & $-49.9 \_-18.9$ & $y=-0.966 x-4.8749$ \\
& $(31-1)$ & $5.4 \_6.1$ & $y=-0.685 x+0.1685$ \\
& $(1-3)$ & $8.1 \_2.9$ & $y=-0.6085 x+3.0882$ \\
& $(23-25)$ & $-37.5 \_6.7$ & $y=-0.5403 x+2.927$ \\
& $(13-15)$ & $-3.2 \_7.0$ & $y=-0.3501 x-10.488$ \\
& $(19-21)$ & $21.9 \_1.1$ & $y=-0.1724 x+3.1707$ \\
& $(29-31)$ & $-1.4 \_2.4$ & $y=-0.1673 x-5.2356$ \\
& $(5-7)$ & $8.8 \_-9$ & $y=-0.0866 x+5.536$ \\
& $(21-23)$ & $11.8 \_-14.9$ & $y=-0.0564 x+6.3962$ \\
\hline stage 3 & $(17-19)$ & $4.3 \_12.6$ & $y=0.4158 x-5.6201$ \\
\hline
\end{tabular}

Extreme unilateral atherosclerosis

$\mathrm{VWd}$

\begin{tabular}{llll}
\hline stage 2 & $(5-7)$ & $6.7 \_-4.3$ & $y=-2.403 x+16.795$ \\
& $(9-11)$ & $11.4 \_-1.3$ & $y=-2.2349 x-2.0211$ \\
& $(1-3)$ & $4.6 \_-0.3$ & $y=-1.464 x+5.0858$ \\
& $(7-9)$ & $-81 \_33$ & $y=-0.8929 x-2.203$ \\
& $(15-17)$ & $0 \_22.3$ & $y=-0.6133 x-4.692$
\end{tabular}




\begin{tabular}{llll} 
& $(11-13)$ & $-7.4 \_7.3$ & $y=-0.5475 x+2.9391$ \\
& $(19-21)$ & $11.5 \_10.8$ & $y=-0.3669 x-3.3153$ \\
& $(3-5)$ & $9.9 \_36$ & $y=-0.3564 x+12.252$ \\
& $(31-1)$ & $-9.4 \_-10.5$ & $y=-0.2104 x+0.0782$ \\
& $(29-31)$ & $-13.9 \_-6.2$ & $y=-0.1912 x-0.2728$ \\
& $(23-25)$ & $11.1 \_5.7$ & $y=0.2662 x-4.7889$ \\
\hline stage 3 & $(27-29)$ & $-21.6 \_-3.5$ & $y=0.2776 x+3.2276$ \\
& $(13-15)$ & $7.2 \_-20.3$ & $y=0.4474 x-3.1283$ \\
& $(21-23)$ & $-0.6 \_18.9$ & $y=0.4833 x-5.4384$ \\
& $(25-27)$ & $2.7 \_10.7$ & $y=0.5251 x-4.5842$ \\
& $(17-19)$ & $11.1 \_20$ & $y=0.8697 x-26.254$
\end{tabular}

Triplets are arranged in descending order based on trendline a-values $\Delta \mathrm{R} l \mathrm{u}-\mathrm{VWd}$.

\section{Discussion}

263

264

265

Excision of pressure perfused vessels is a standard method used to prepare and examine the cardiac vessels at autopsy because it allows better approximation of the invivo lumen and vessel wall configuration. However, no agreement has been reached on the best post-mortem vessel examination technique, with wide ranges of perfusion pressures used in different studies making it impossible to compare results. Here, we used non-perfused vessels to better preserve the elastic characteristic of the coronary vessel wall, with residual wall stress clearly evident by the cut ends of the walls moving away from each other after longitudinal incision. Sections cut perpendicular to the longitudinal axis maintain residual wall stress, thereby retaining the basal lumen and vessel wall configuration [7]. This approach preserves the mutual relationships, as confirmed by our results.

In essence, the main basic process is the 'counter-balancing' system: growth of $\Delta \mathrm{INTd}$, $\triangle \mathrm{MEDd}$, or $\triangle \mathrm{ADVd}$ counter-balanced by the other two. As such, these are functionally united in a direct way $(\Delta \mathrm{FU}-1)$. This basic function stays intact regardless of vessel wall pathology, though triplets present the same sign in a minority of sections. In an indirect way $\Delta \mathrm{INTd}$, 
$\triangle \mathrm{MEDd}$, and $\triangle \mathrm{ADVd}$ determine the course of $\triangle \mathrm{VWd}$ in every triplet (increasing or decreasing), its measure by semi-range values and their sign change.

Within each section, stages are defined in which the mutual relationship between $\Delta \mathrm{VWd}$ and $\Delta$ Rlu changes, from an increase in $\Delta \mathrm{VWd}$ and increased $\Delta \mathrm{Rlu}$ (stage 1 and stage 3 ) to a decrease in $\Delta \mathrm{Rlu}$ and increased $\Delta \mathrm{VWd}$ (stage 2). The transition of stage 1 to stage 2 is marked by trendline a-value $\Delta \mathrm{VWd}=0$ and $\Delta \mathrm{Rlu}=1$, whereas the transition from stage 2 to stage 3 is marked by a-value $\Delta \mathrm{VWd}=1$ and $\Delta \mathrm{Rlu}=0$. The a-value $\Delta \mathrm{VWd}=0$ and $\Delta \mathrm{Rlu}=0$ reflect the peak/bottom parabola values before data transition to $\Delta$ values, which transforms a parabolic course to a linear course. As such, stages are defined by the $\Delta \mathrm{VWd}$ and $\Delta \mathrm{Rlu}$ parabolic course remnants, a change from synchronic (same trendline a-value sign, stages 1 and 3) to asynchronic (opposite trendline sign, stage 2). This proves that the $\Delta \mathrm{VWd} / \Delta \mathrm{Rlu}$ relationship is course- and sign-dependent and, therefore, independent of their values in absolute terms.

Atherosclerotic and non-atherosclerotic sections cannot be differentiated by triplet behavior. For example, $\Delta \mathrm{INTd}$ growth with atherosclerosis is marked only by a shift from stage 1 to stage 3. This illustrates that even atherosclerotic sections exhibit an increase in $\Delta \mathrm{Rlu}$ in relation to $\Delta \mathrm{VWd}$ growth, which implies independence from vessel wall constitution. Support for this view is given by the summed semi-range values $\triangle \mathrm{INTd}, \triangle \mathrm{MEDd}$, and $\triangle \mathrm{ADVd}$ in proportion to semi-range $\Delta \mathrm{Rlu}$, which is similar to the $\Delta \mathrm{VWd} / \Delta \mathrm{Rlu}$ ratio in absolute terms. Therefore, the atherosclerotic coronary vessel wall cannot be considered inert, but still proves to be a well-adapting 'organ'.

The $\Delta$ Rlu course is entirely dependent on the $\Delta \mathrm{VWd}$ course, but their relationship is only consistent within each triplet and, as such, variable between different triplets. Consequently, the $\Delta \mathrm{VWd}$ has a dissimilar course within each singular triplet, indicating that the quest for a 
specific coronary vessel wall dimension as parameter of 'physiological' or 'pathological' is futile. The same is valid for the intima, media, and adventitia dimensions. Both coursedetermining factors prove the existence of a strict control mechanism active within all sections and un-altered by the atherosclerotic process.

Successive events describe a system's course, which is artificial in regards to non-consecutive triplets within each section. This added to the finding that consecutive sections resemble each other only roughly (not shown), assuming systems processes are active throughout the vessel wall. If and how the control mechanism functions in a longitudinal way should be revealed by further investigation.

Overall, the findings indicate that basic systems and mechanisms are un-altered by the atherosclerotic process, which makes a systemic defect as the cause of atherosclerosis less probable and a superposed agent (e.g., inflammation) more plausible. The constituent parts of the coronary vessel wall (intima, media, adventitia) act as a true functional unit via a counterbalancing system and cannot be considered as representative of the coronary vessel wall pathology. If this is also the case with elastic arteries, such as the carotid arteries, using the intima/media ratio as a parameter of coronary artery pathology could be debatable $[8,9]$. The stages observed in every section could instigate reconsideration of the cause of coronary artery remodeling [10].

Atherosclerosis is an umbrella term for a process in which plaques are ultimately formed. The aorta appears more ulcerative (craters) than the coronary arteries and the processes leading to these differences uncertain. Therefore, findings on coronary atherosclerotic processes cannot be considered normative for the aortic processes. Differences could exist between muscular and elastic arteries, or even between different muscular or elastic arteries. This is illustrated 
by the coronary arteries initially lacking the sub-endothelial (intimal) layer, in contrast to cerebral arteries initially lacking the adventitial layer, making them translucent in vivo.

Therefore, our study is only the first step in understanding the process from arterial physiology to pathology. Further investigation of different arteries (single sections and section series) could elucidate similarities and/or differences. The random systemic sampling technique could aid in obtaining results for comparisons.

\section{Conflict of interest: none}

\section{References}

1. Stary HC, Chandler AB, Dinsmore RE, Fuster V, Glagov S, Insull W, et al. A definition of advanced types of atherosclerotic lesions and a histological classification of atherosclerosis. A report from the Committee on Vascular Lesions of the Council on Arteriosclerosis, American Heart Association. Circulation. 1995;92: 1355-1374.

2. Stary HC, Chandler AB, Glagov S, Guyton JR, Insull W, Rosenfeld ME, et al. A definition of initial, fatty streak, and intermediate lesions of atherosclerosis. A report from the Committee an Vascular Lesions of the Council on Arteriosclerosis, American Heart Association. Arterioscler Thromb Vasc Biol. 1994;14: 840-856.

3. Herrmann J, Lerman A. Coronary artery disease: development and progression. In: Barsness GW, Holmes DR, editors. Coronary artery disease. London: Springer; 2012. pp. 2128.

4. Stary HC, Blankenhorn DH, Chandler AB, Glagov S, Insull W, Richardson M, et al. A definition of the intima of human arteries and its atherosclerosis-prone regions. A report from 
the Committee on Vascular Lesions of the Council on Arteriosclerosis, American Heart

347 Association. Circulation. 1992;85: 391-405.

348 5. Schwartz SM, Blois de D, O’Brien RM. The Intima: Soil for atherosclerosis and 349 restenosis. Circ Res. 1995;77: 445-465.

350 6. De Groot P, Veldhuizen RW. Human coronary artery remodeling, beginning and end 351 of the atherosclerotic process. PLoS One. 2006;1: e91.

352 7. Humphrey JD. Cardiovascular solid mechanics, cells, tissues, and organs. London:

353 Springer; 2010.

354 8. Inaba Y, Chen JA, Bergmann SR. Carotid plaque, compared with intima-media

355 thickness, more accurately predicts coronary artery disease events: A meta-analysis.

356 Atherosclerosis. 2012;220: 128-133.

357 9. Amato M, Montorsi P, Ravani A, OldaniStefano E, et al. Carotid intima-media

358 thickness by B-mode ultrasound as surrogate of coronary atherosclerosis: correlation with

359 quantitative coronary angiography and intravascular ultrasound findings. Eur Heart J.

$360 \quad 2007 ; 28: 2049-2050$.

361 10. Glagov S, Weisenberg E, Zarins CK, et al. Compensatory enlargement of human

362 atherosclerotic coronary arteries. New Engl J Med. 1987;316: 1371-1375. 


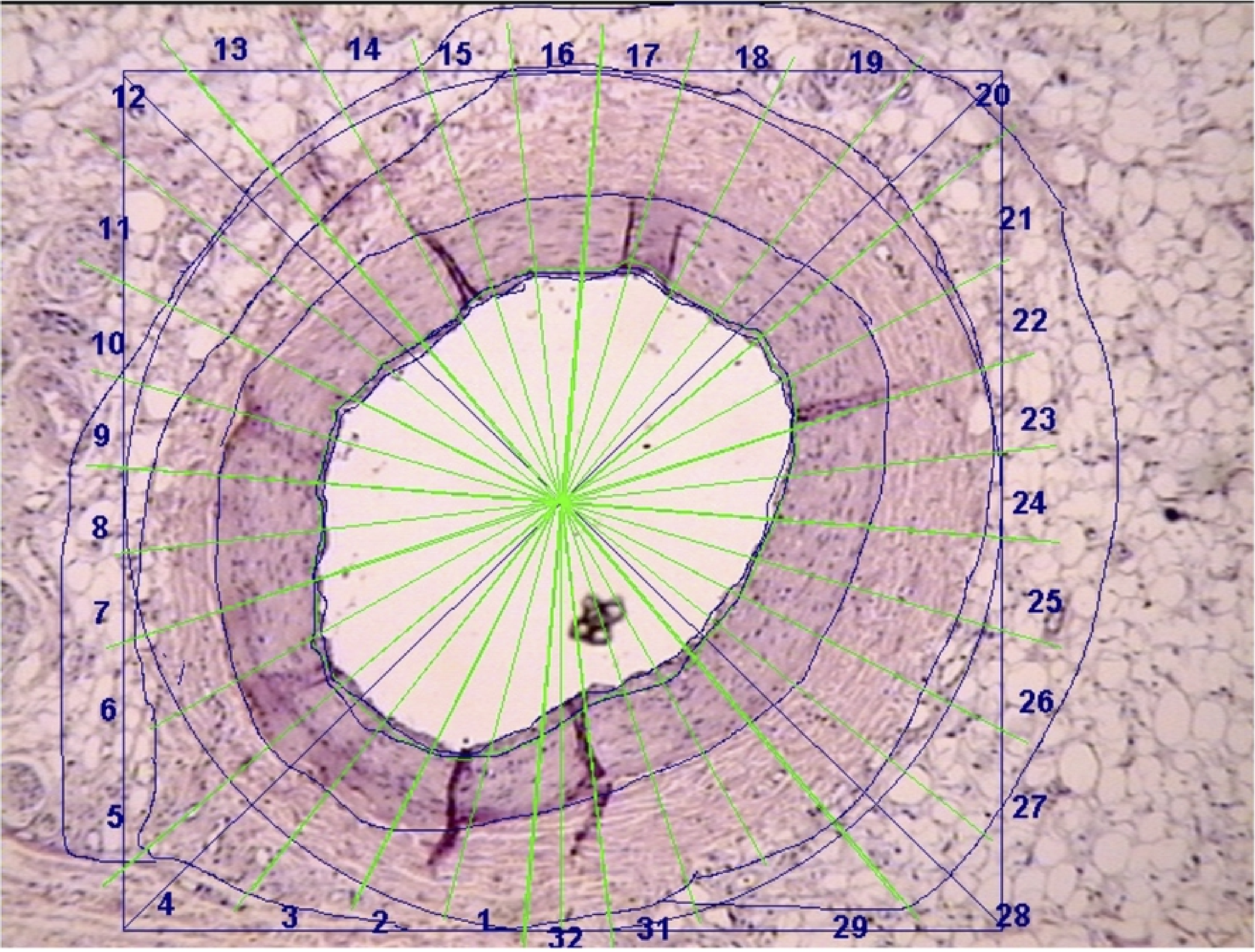

Figure 


\section{A}

S427/85, cor 5-1

EvG, obj.2,5

doos $70, \mathrm{nr} .56$

bioRxiv preprint doi: https://doi.org/10.1101/581454; this version posted March 19, 2019. The copyright holder for this preprint (which was not certified by peer review) is the author/funder, who has granted bioRxiv a license to display the preprint in perpetuity. It is made

B

\section{Section "normal".}

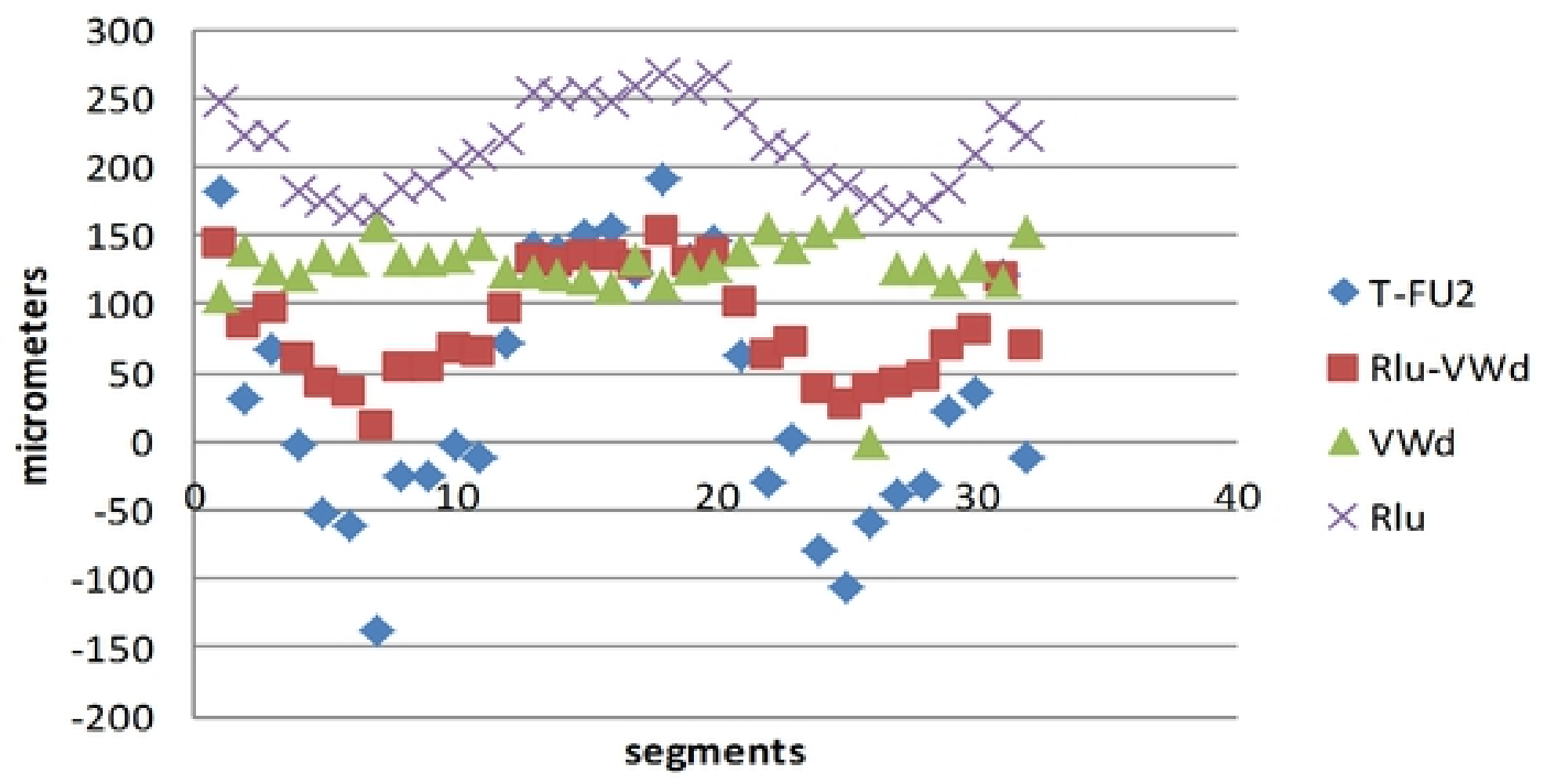

C

\section{Section "normal".}

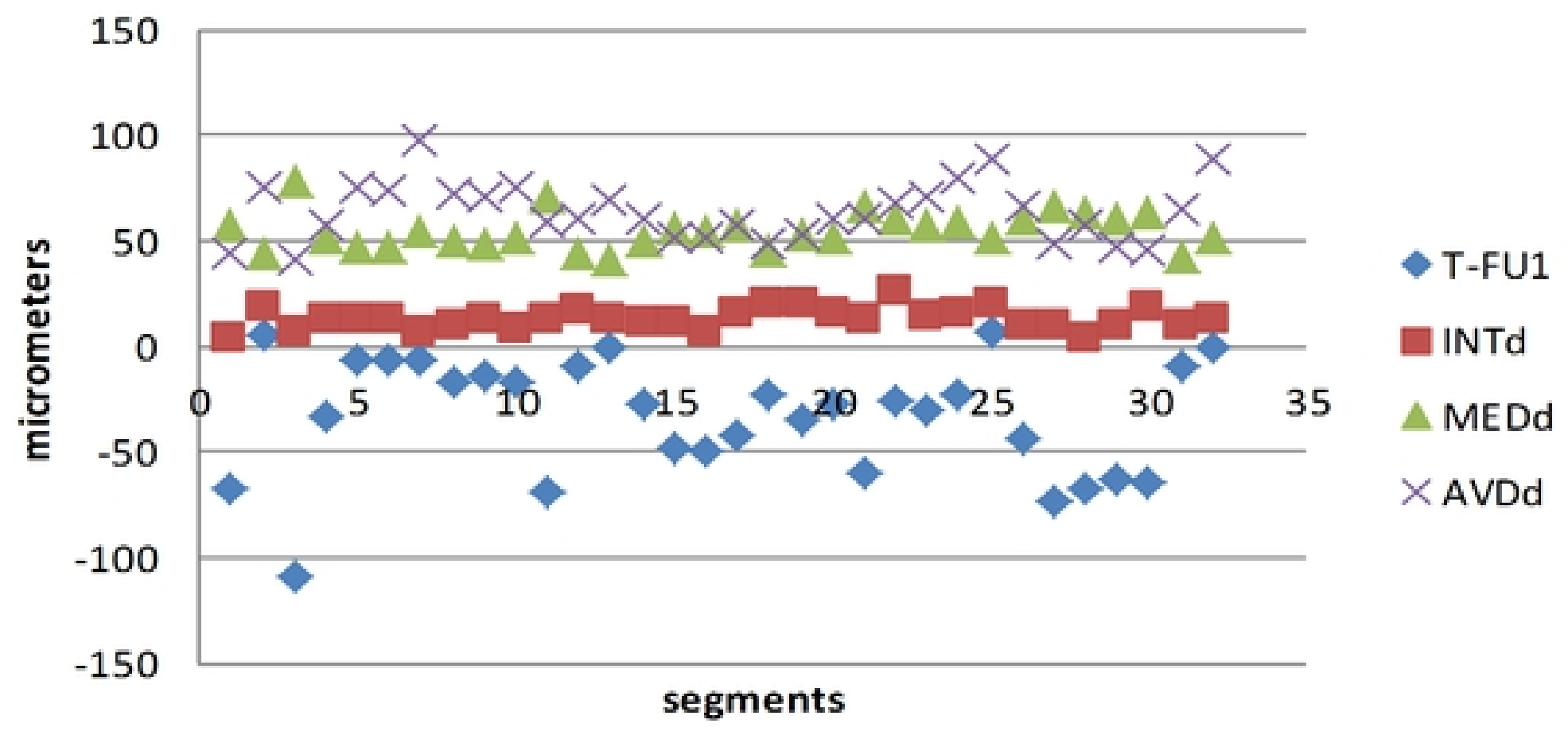

\section{Figure}




\section{A}

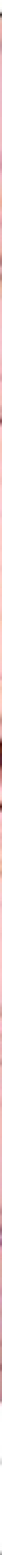

\section{Section "circumferential atherosclerosis"}

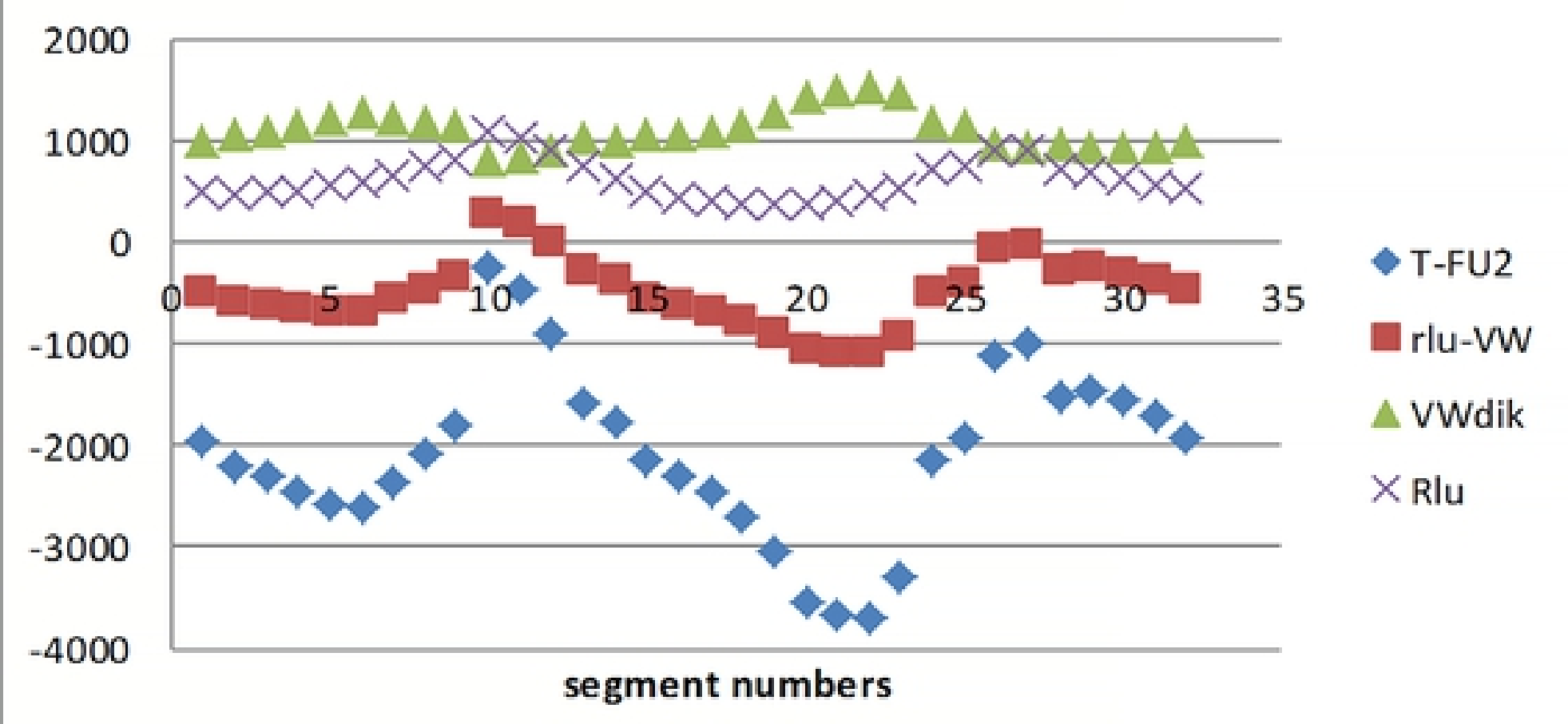

\section{C}

\section{Section "circumferential atherosclerosis".}

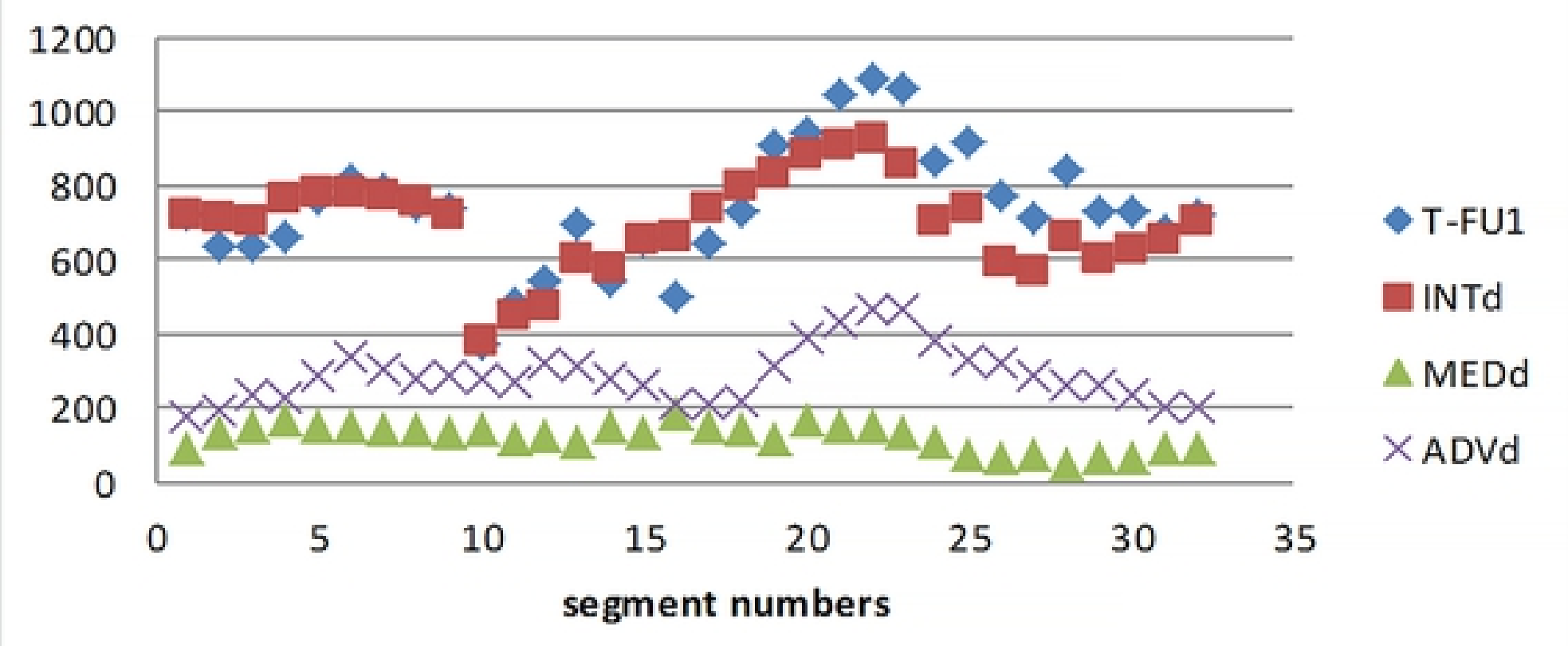

Figure 